\title{
Outsourcing Private RAM Computation
}

\author{
Craig Gentry* $^{*} \quad$ Shai Halevi ${ }^{\dagger} \quad$ Mariana Raykova ${ }^{\ddagger} \quad$ Daniel Wichs ${ }^{\S}$
}

August 25, 2014

\begin{abstract}
We construct the first schemes that allow a client to privately outsource arbitrary program executions to a remote server while ensuring that: (I) the client's work is small and essentially independent of the complexity of the computation being outsourced, and (II) the server's work is only proportional to the run-time of the computation on a random access machine (RAM), rather than its potentially much larger circuit size. Furthermore, our solutions are non-interactive and have the structure of reusable garbled RAM programs, addressing an open question of $\mathrm{Lu}$ and Ostrovsky (Eurocrypt 2013). We also construct schemes for an augmented variant of the above scenario, where the client can initially outsource a large private and persistent database to the server, and later outsource arbitrary program executions with read/write access to this database.

Our solutions are built from non-reusable garbled $R A M$ in conjunction with new types of reusable garbled circuits that are more efficient than prior solutions but only satisfy weaker security. For the basic setting without a persistent database, we can instantiate the required type of reusable garbled circuits from indistinguishability obfuscation or from functional encryption for circuits as a black-box. For the more complex setting with a persistent database, we can instantiate the required type of reusable garbled circuits using stronger notions of obfuscation. It remains an open problem to instantiate these new types of reusable garbled circuits under weaker assumptions, possibly avoiding obfuscation altogether.

We also give several extensions of our results and techniques to achieve: schemes with efficiency proportional to the input-specific RAM run-time, verifiable outsourced RAM computation, functional encryption for RAMs, and a candidate obfuscator for RAMs.
\end{abstract}

\section{Introduction}

Outsourcing computation from a weak client to a more powerful server is quickly becoming the predominant mode of day-to-day computation, bringing with it new security challenges and flourishing research into methods for addressing them. In this work we consider the challenge of private outsourcing, where the client wants to execute a program on a remote server while hiding from it the raw data to be used in the computation. Moreover, we want to ensure that:

1. The client should perform significantly less work than executing the program, and

2. The server should not have to do much more work than executing the program. One method of outsourcing computation relies on fully homomorphic encryption (FHE) [RAD78, Gen09], where the client simply encrypts her input and decrypts the output, and the server computes the program on encrypted data. Unfortunately, this solution requires the server to translate the program into a circuit and therefore work as hard as the circuit size of the computation, which

\footnotetext{
*IBM Research, T.J. Watson. E-mail: cbgentry@us.ibm.com.

${ }^{\dagger}$ IBM Research, T.J. Watson. E-mail: shaih@alum.mit.edu.

${ }^{\ddagger}$ SRI. E-mail: mariana@cs. columbia.edu. Research conducted in part while at IBM Research.

$\S$ Northeastern University. E-mail: wichs@ccs.neu.edu. Research conducted in part while visiting IBM Research. Supported in part by NSF grant 1347350.
} 
in general, can be much larger than the work needed to execute the program on a random-access machine (RAM). In particular, even if we reach the zenith of FHE efficiency, with no overhead per homomorphic addition/multiplication, simply converting the computation into a circuit may already be too inefficient.

In general, a RAM computation with run-time $t$ can have Turing-Machine run-time and circuit size as high as $\widetilde{O}\left(t^{2}\right)$, which is already a considerably large overhead [CR73, PF79]. However, this only applies to computations where the memory starts out empty, and the gap can be significantly larger in a setting involving program executions over large data stored in memory (e.g., a database). Consider for example the setting of private information retrieval (PIR) [CKGS98], where a server holds a large database of size $N$ and a client wants to simply retrieve a single record from that database without the server learning the requested record. In this case, the RAM complexity of the retrieval query can be as low as $O(\log N)$, but the circuit complexity must be $\Omega(N)$ since the circuit must at least get the entire database as input. This a fully exponential gap can mean the difference between and efficient Internet search and reading the entire Internet. The same gaps also already appear if we were to consider the Turing-Machine run-time of the computation instead of its circuit size.

We therefore would like to find an outsourcing protocol in which the server's work is only related to the RAM complexity of the program, while the client's work is essentially independent of the complexity of the program altogether. Furthermore, we would like to have such protocols in a setting where the client can initially outsource some persistent memory data (e.g., containing a database) and later outsource various RAM computations with read/write access to this memory.

Prior to this work, no such protocols were known. Although we do have private computation protocols over an outsourced memory based on oblivious RAM (ORAM) (e.g., [GO96, OS97, $\mathrm{GKK}^{+} 12$, LO13a, $\left.\mathrm{GHL}^{+} 14\right]$ ) where the server's work is proportional only to the RAM complexity of the computation, in all of these protocols the client also works as hard as the server. In particular, these protocols allow the client to save on storage by outsourcing the data to a remote server, but they do not provide any savings in computation over executing the program locally on local data.

In this work we describe reusable garbled $R A M$ schemes, which offer the first solution to private outsourcing of RAM computation, where the server's work is only proportional to the RAM runtime of the computation and the client's work is essentially independent of the complexity of the computation altogether. In addition, these protocols are non-interactive, i.e., they only use one-way communication if the server is to learn the output (or two message communication if the client is to learn the output), making them useful even beyond outsourcing in the "send and forget" settings.

\subsection{Garbled Circuits and Garbled RAM}

(Reusable) Garbled Circuits. Garbled circuits, introduced in the seminal work of Yao [Yao82], allow a client to garble a circuit $C$ and then an input $x$ in such a way that a server can use these garbled values to compute $C(x)$ without learning anything more about $x$. Until recently, all such known schemes became insecure if the server ever got to see more than one garbled input per garbled circuit. In particular, such schemes are not very useful in the context of outsourcing computation, since the client would have to create a fresh garbled circuit for each computation and therefore perform work proportional to the circuit size. Last year Goldwasser et al. described the first reusable circuit-garbling scheme $\left[\mathrm{GKP}^{+} 13 \mathrm{~b}\right]$ where the client can garble a single circuit and then garble many inputs to that circuit without losing security. This allows private outsourcing of circuit computation where the client only needs to do a one-time pre-processing step to garble the circuit, at a cost proportional to the circuit size. The client can then outsource many computations of this circuit on many different inputs by garbling them, with essentially no additional work per 
computation beyond what is needed to send the input.

Reusable Garbled TMs. The work of Goldwasser et al. $\left[\mathrm{GKP}^{+} 13 \mathrm{a}\right]$ extends the notion of reusable gabled circuits to Turing Machines (TMs). The main advantage is that the work of garbling a TM and the size of the garbled TM can be made proportional to the TM description size rather than the larger TM run-time or circuit size of the computation. In the context of outsourcing computation, this translates to getting rid of the pre-processing step so that the client never has to work as hard as evaluating the program. Another advantage of TMs over circuits is that TM computation can have much smaller "per-instance run-time" on some inputs than its "worst-case run-time". The solution of $\left[\mathrm{GKP}^{+} 13 \mathrm{a}\right]$ allows the server to run in time proportional to the per-instance TM run-time of the computation, at the security loss of leaking this run-time to the server. These advantages are mainly orthogonal to our main goal of allowing the server to work in time proportional to the RAM run-time of the computation, which could potentially be much smaller than the TM run-time or the circuit size. For example, when outsourcing a binary search over a large outsourced database of size $N$, both the circuit-size and the per-instance TM run-time will be $O(N)$ whereas the worst-case RAM run-time is $O(\log N){ }^{1}$

Garbled RAM. Also recently, Lu and Ostrovsky introduced the notion of garbled RAM [LO13a]. Similar to garbled circuits, the client can garble a RAM program $P$, and later garble an input $x$ in such a way that a server can use these garbled values to compute $P(x)$ without learning anything more about $x$. The complexity of garbling a RAM program (client complexity), the size of the garbled RAM, and the complexity of evaluating a garbled RAM (server complexity) are all proportional to the RAM run-time of the program rather than its circuit size. ${ }^{2}$ The constructions of garbled RAM uses a clever combination of Yao garbled circuits and oblivious RAM (ORAM). Just like in Yao's circuits, the scheme is not reusable and becomes completely insecure if the server sees more than a single garbled input per garbled program. In other words, the client has to garble a fresh program for every computation, which requires as much work as doing the computation and therefore does not offer any savings in the context of outsourcing.

However, garbled RAM does offer an opportunity for amortization in the more complex setting involving multiple program executions over some persistent memory (e.g., a large outsourced database). The client can garble the initial memory contents once, and then can garble many different RAM programs and inputs (one input per program) that would be executed relative to the garbled memory, updating the memory with every execution. This property is called persistent memory, and allows the garbled memory to be reused. For example, the client can garble a large database of size $N$ only once in time $O(N)$, and after that garble arbitrary queries to the database where the client work (time to garble a query) and the server work (time to evaluate a garbled query) are both proportional to the RAM run-time of the query. This provides a good solution for cases where the memory is large and the client wants to save on storage by outsourcing the contents, but the database queries are sufficiently simple that the client does not mind doing the work of the computation. It improves on simply using ORAM by making the program executions completely non-interactive. Nevertheless, it still provides no savings in terms of client computation over having the client store the data and perform all computations locally.

\footnotetext{
${ }^{1}$ Although we mainly focus on our primary goal of having the server work in time proportional to the worst-case RAM run-time, we will also show a simple extension that reduces this to the per-instance RAM run-time using the techniques of $\left[\mathrm{GKP}^{+} 13 \mathrm{~b}, \mathrm{GKP}^{+} 13 \mathrm{a}\right]$.

${ }^{2}$ It was recently observed that the security proof for the scheme of [LO13a] has a subtle flaw, but the scheme can be fixed so as to get essentially the same properties as the original scheme $\left[\mathrm{GHL}^{+} 14\right]$.
} 
Can Garbled RAM be Reusable? The above raises the natural question whether we can obtain a reusable garbled RAM. In such a scheme, the client can garble a program once as a potentially expensive pre-processing step, and later outsource many arbitrary computations of this program to a server by efficiently garbling fresh inputs. The server can evaluate the garbled program on a garbled input in time proportional to the RAM complexity of the program. Furthermore, we would also like to do this in a setting where the client initially garbles a large persistent memory (e.g., database) and the programs can read/write to this memory. Such reusable garbled RAM schemes give a particularly nice solution to the problem of outsourcing private RAM computation with no interaction in the case where the server is to learn the output, and one round of back-andforth communication when the client is to learn the output. The output can be made private from the server by simply garbling an augmented program that returns the output encrypted under the client's key. Furthermore, it can be made verifiable so that the client can be sure that the received output is correct, by simply garbling an augmented program that returns the output along with a message-authentication-tag of the output, under a key provided with the input. ${ }^{3}$

\subsection{Our Solutions}

In this work we describe the first solutions to the above problem of reusable garbled-RAM, with various tradeoffs between features/efficiency and the security assumptions needed to instantiate them.

As our "basic" solution, we describe a protocol that works in the setting without persistent memory, and requires the client to perform an expensive one-time pre-processing step to garble the program. In particular, the client can take a RAM program $P$ along with some bound $t$ on its run time and create a garbled version by working in time $\widetilde{O}(t)$. It can then very efficiently garble arbitrarily many inputs $x_{j}$ to that program in time only proportional to the input (and output) size of the program, but independent of the run-time $t$. The server can evaluate the garbled program on each garbled input in time $\widetilde{O}(t)$. Furthermore, garbling new inputs only requires a public key, so anybody can outsource computations by creating garbled inputs to the garbled program. ${ }^{4}$

As our "best-case" solution, we describe a protocol that also works in the more complex setting involving persistent memory (e.g., database) and does not require any expensive pre-processing. Specifically, in this solution the client has the option to garble some persistent memory of size $N$ in time $\widetilde{O}(N)$. It can then garble a RAM program $P$ in time proportional to its description length $|P|$ but independent of its running time. Finally it can garble many "short inputs" $x_{i}$ to the program $P$ in time proportional to the input (and output) size of the program. The server can evaluate the garbled program with the garbled input over the garbled memory in time proportional to the program's RAM run-time $\widetilde{O}(t)$. For example, the programs $P$ could be a SQL database implementation and the inputs $x_{i}$ could specify various complex database queries. We stress that the program executions can both read and write to memory, and that the changes to memory made by one program execution persist for the next program execution and cannot be "rolled back" by a malicious server.

\footnotetext{
${ }^{3}$ We note that there are other approaches to verifiable RAM computation using SNARKs and proof-carrying data [Val08, BCCT13, BSCGT13, BSCG $\left.{ }^{+} 13, \mathrm{BFR}^{+} 13\right]$, but no other prior approaches that provide privacy. Therefore, we view the question of privacy as more pressing, but note that reusable garbled RAM gives us verifiability for free.

${ }^{4}$ By default, we do not require "program privacy" and allow the server to learn the description of the outsourced program. In the public-key setting this is inherent since the server can create garbled inputs on his own and therefore learn information about the functionality of the program. We only guarantee that the server does not learn anything about the inputs that are garbled by the client, beyond the output of the computation. We will describe a simple extension that achieves program privacy, but necessarily moves the construction to the secret key setting where only the client that creates the garbled program can create garbled inputs.
} 
Assumptions. Our main contribution is to reduce the complex problems of reusable garbled $R A M$ to seemingly simpler problems dealing with reusable garbled circuits, and avoiding the complexity of RAM altogether. The above constructions correspond to new notions of security/efficiency for reusable garbled circuits, which may be of independent interest (see below). Ultimately, we can instantiate these new notions of reusable garbled circuits using various obfuscationbased assumptions. The garbled circuits that are used in our "basic" solution can be based on indistinguishability obfuscation or on the existence of indistinguishability-secure functional encryption for circuits. In particular, the latter is a falsifiable assumption. The reusable garbled circuits needed for our "best-case" solution can be based on stronger variants of obfuscation, related to differing-inputs obfuscation. We stress that the use of obfuscation does not seem inherent, and there is hope that these new notions of reusable garbled circuits could be instantiated under simpler assumptions that avoid obfuscation altogether.

\subsection{Our Techniques}

We obtain reusable garbled RAM from a combination of non-reusable garbled RAM, and a new form of reusable garbled circuits whose properties we discuss shortly.

Our solutions are based on a very simple intuitive idea: given a RAM program $P$, consider the circuit $C[P]$ which has $P$ hard-coded in its description, gets as input $(r, x)$, and uses $r$ as randomness to created a one-time garbled program $\tilde{P}_{\text {one }}$ (garbling $P$ ) and a garbled input $\tilde{x}_{\text {one }}$ (garbling $x$ ). Garbled RAM ensures that the circuit-size of $C[P]$ is only dependent on the RAM run-time $t$ of the program $P$ rather than its potentially much larger circuit size. Our main idea is to create a reusable garbled circuit $\tilde{C}_{\text {reuse }}$ of the circuit $C[P]$, which the client gives to the server. Each time the client wants to run a new program execution with input $x_{i}$, she chooses some fresh randomness $r_{i}$, and garbles $\left(r_{i}, x_{i}\right)$ under the reusable circuit garbling scheme. The server runs the reusable garbled circuit $\tilde{C}_{\text {reuse }}$ on the garbled input from the client to create a one-time garbled RAM program $\tilde{P}_{\text {one }}$ and garbled input $\tilde{x}_{\text {one }}$, and then evaluates $\tilde{P}_{\text {one }}$ on $\tilde{x}_{\text {one }}$.

The above idea is not entirely new, but it turns out that it cannot quite work right out of the box. ${ }^{5}$ Notice that the circuit $C[P]$ above has a huge output of size $\widetilde{O}(t)$ even though its input is small. Unfortunately, the reusable garbled circuit construction of Goldwasser et al. [GKP $\left.{ }^{+} 13 \mathrm{~b}\right]$, requires that the size of the garbled input to the circuit always exceeds the size of the circuit's output, even if the size of the actual input of the circuit is small. ${ }^{6}$ We call this property outputsize dependence. In particular, to securely garble a short input $\left(r_{i}, x_{i}\right)$, the client would have to create a huge garbled input of size $\widetilde{O}(t)$, which would require that the client works at least as hard as evaluating the program, and completely obliterate the efficiency benefits of outsourcing. Unfortunately, this is also not an accidental property of the construction of $\left[\mathrm{GKP}^{+} 13 \mathrm{~b}\right]$ and we show that any reusable circuit garbling scheme with simulation-based security must have outputsize dependence (see Appendix C).

Our main observation is that we do not necessarily require full simulation-based security from the reusable garbled circuit component, even though we insist on achieving full simulation-based security for the final reusable garbled RAM construction. We come up with new notions of security for reusable garbled circuits that we call "distributional indistinguishability" (with two flavors), which turn out to suffice in our constructions and may be of independent interest elsewhere. Intuitively, these notions say that one cannot distinguish garbled inputs from two distributions that produce indistinguishable outputs. Moreover, these weaker security notions seem to plausibly allow

\footnotetext{
${ }^{5} \mathrm{~A}$ variant of an idea along these lines appeared in an early version of [LO13a] and was outlined in a rump-session talk [LO13b] but was retracted for exactly the reasons we describe here.

${ }^{6}$ The scheme and parameters of $\left[\mathrm{GKP}^{+} 13 \mathrm{~b}\right]$ are described for circuits with 1-bit output, but can easily be extended to the setting of multi-bit output at the above cost of having the size of the garbled-input grow with the output size.
} 
for more efficient constructions that avoid "output-size dependence". Indeed, we propose new candidate constructions of such reusable garbled circuits based on obfuscation. Our two constructions of reusable garbled RAM translate to two flavors of reusable garbled circuits with "distributional indistinguishability". The weaker flavor can be based on indistinguishability obfuscation while the stronger one seems to require stronger obfuscation-based assumptions. It remains as an open problem to achieve these notions from other assumptions, ideally avoiding obfuscation altogether.

\subsection{Extensions}

In Section 6 we explore several extensions and applications of our main results and techniques. We discuss how to generically augment reusable garbled RAM to get output privacy (server does not learn the output of the computation) and verifiability (client can be certain that the received output is correct). We also discuss how to get program privacy where the server does not learn the code of the program. Furthermore, we discuss how to leverage our solutions to get input-specific run-time where the server's work is only proportional to the RAM run-time of $P(x)$ on the desired input $x$ rather than the worst-case run-time of $P$ on inputs of size $n$. We also discuss applications to MPC where only one party needs to work as hard as the program's RAM run-time. Then in Section D, we show how to leverage our techniques to build indistinguishability-secure functional encryption (FE) for RAM programs (without persistent data) using FE for circuits as a black box. We show that this also gives an alternate construction of reusable garbled RAM from FE for circuits, without using obfuscation directly. However, the only known construction of FE for circuits in $\left[\mathrm{GGH}^{+} 13 \mathrm{~b}\right]$ relies on iO. Lastly, in Section E, we propose a speculative candidate construction for obfuscating RAMs using obfuscation for circuits and conjecture that it can achieve iO for RAMs.

Concurrent Work. A concurrent and independent work of Apon et al. $\left[\mathrm{AFK}^{+} 14\right]$ achieves similar results on outsourcing RAM computation, as well as functional encryption for RAMs. Appendix D of this work, showing functional encryption for RAMs, was added after the work of Apon et al. was posted. This appendix relies on simple extensions of our main ideas for reusable garbled RAM, which we view as our main contribution.

\section{Preliminaries}

The two models of computation that we deal with in this work are circuits and RAM programs. Intuitively, a RAM program has access to some memory of size $N$ and each step of the program can read/write to an arbitrary location of memory. We usually assume that the memory starts out empty. However, when we consider program executions over a persistent memory/database, it is useful to consider the case where the memory initially contains some data $D$. We use the notation $P^{D}(x)$ to denote the execution of a program $P$ with random-access memory containing $D$ and a short input $x$. For the RAM programs we consider in this work, we assume that we have an absolute bound on their worst-case running time, input/output length, and memory usage. A somewhat more detailed specification of the RAM model is found in Appendix B.1.

We use $C[\mathrm{prm}]$ or $P[\mathrm{prm}]$ to denote a circuit/program that depends on a parameter prm. The parameter can be an arbitrary string, and can itself be another circuit or program. We think of prm as being "hard wired" in the description of the corresponding circuit/program. The input to

a circuit/program is specified inside parenthesis, so $C[\mathrm{prm}](x)$ describes the computation of the circuit $C[\mathrm{prm}]$ (whose definition depends on prm) on the input $x$. 


\section{Reusable GRAM without Persistent Memory}

\subsection{Defining Garbled RAM}

We begin by defining non-reusable (one-time) and reusable garbled RAM. The syntax of the scheme is the same in both cases, and the difference is only in the security requirements.

Definition 3.1 (GRAM). A garbled RAM scheme (without persistent memory) consists of procedures $\mathrm{GR}=$ (GR.prog, GR.inp, GR.eval):

- $(\tilde{P}, s) \leftarrow G R \cdot \operatorname{prog}\left(1^{\lambda}, P,(n, m, t)\right):$ Gets a RAM program $P$, and bounds on the program's: input size $n$, output size $m$, and run-time $t$ (say that all bounds encoded in binary). Outputs a garbled program $\tilde{P}$ and a garbling key $s$.

- $\tilde{x} \leftarrow \operatorname{GR} \operatorname{inp}(x, s,(n, m, t)):$ Takes as input an $n$-bit value $x$, the garbling key s the same bounds $(n, m, t)$. It outputs the garbled input $\tilde{x}$.

- $y=\operatorname{GR} \cdot \operatorname{eval}(\tilde{P}, \tilde{x})$ : This is a RAM program that takes $(\tilde{P}, \tilde{x})$ as input and computes the output $y$.

We require that for any program $P$ with parameters $(n, m, t)$, any input $x \in\{0,1\}^{n}$ if $\tilde{P}, \tilde{x}$ are created as described, then $\mathrm{GR} \cdot \operatorname{eval}(\tilde{P}, \tilde{x})=P(x)$ with probability 1 .

Definition 3.2 (GRAM Security). Let GR be a garbled RAM scheme as above.

- GR has reusable security if there exists a PPT simulator Sim such that, for all RAM programs $P$ with polynomial parameters $(n, m, t)$ and all polynomial-length input-vectors $\left(x_{1}, \ldots, x_{q}\right)$, the following distributions are computationally indistinguishable:

$$
\left(\tilde{P}, \tilde{x}_{1}, \ldots, \tilde{x}_{q}\right) \stackrel{\operatorname{comp}}{\approx} \operatorname{Sim}\left(1^{\lambda}, P,(n, m, t), y_{1}, \ldots, y_{q}\right)
$$

where $(\tilde{P}, s) \leftarrow \mathrm{GR} \cdot \operatorname{prog}\left(1^{\lambda}, P,(n, m, t)\right), \tilde{x}_{i} \leftarrow \mathrm{GR} \cdot \operatorname{inp}\left(x_{i}, s,(n, m, t)\right)$ and $y_{i}=P\left(x_{i}\right)$. The simulator is required to run in time poly $(\lambda,|P|, n, m, t, q)$.

- GR has security with public input garbling if there exists a PPT simulator Sim' such that the above security holds even when including the input-garbling key s in the left-hand distribution,

$$
\left(\tilde{P}, s, \tilde{x}_{1}, \ldots, \tilde{x}_{q}\right) \stackrel{\text { comp }}{\approx} \operatorname{Sim}^{\prime}\left(1^{\lambda}, P,(n, m, t), y_{1}, \ldots, y_{q}\right) .
$$

- GR has one-time security (resp. one-time security with public input garbling) if the above only holds for $q=1$.

Efficiency. We will analyze the efficiency of our constructions in Section 5. The main aspect that we will always require is that, for a program $P$ with run-time $t$, the server's run-time in evaluating the garbled program (run-time of GR.eval on a RAM) should only scale with $\widetilde{O}(t)$ and the client's run-time in outsourcing a computation (run-time of GR.inp) should only depend logarithmically on $t$. The run-time of garbling a program (GR.prog) can scale with $\widetilde{O}(t)$ in our "basic" construction, in which case we think of it as a one-time pre-processing, or it can even be independent of $t$ in our "best case" construction. 
One-Time garbled RAM. We rely on prior constructions of one-time secure garbled RAM schemes [LO13a, GHL ${ }^{+}$14]. See Appendix B for an overview of this construction. We state the necessary assumptions and efficiency parameters of the construction in Section 5. The main efficiency property which we will crucially rely on is that the program garbling procedure $(\tilde{P}, s) \leftarrow$ GR.prog $\left(1^{\lambda}, P,(n, m, t)\right)$ in this construction can be expressed as a circuit whose size scales with $\widetilde{O}(t)$. The evaluation of the garbled program $\tilde{P}$ on a garbled input is a $R A M$ computation which should only scale with $\widetilde{O}(t)$.

Remark on Program Privacy. Note that our definition does not explicitly consider program privacy and we assume that the code of the program $P$ is public. This can be fixed via standard transformations, see Section 6.

\subsection{Garbled Circuits}

As a useful tool, we will rely on the notion of reusable garbled circuits and we begin by defining the syntax of such schemes. We defer discussion of the security and efficiency properties that we require from garbled circuits until later.

Definition 3.3 (Garbled Circuits). A garbled circuit scheme consists of three procedures, GC = (GC.circ, GC.inp, GC.eval):

- $(\tilde{C}, s) \leftarrow \mathrm{GC} \cdot \operatorname{circ}\left(1^{\lambda}, C\right):$ Gets an input circuit $C$ and outputs a garbled circuit $\tilde{C}$ and key $s$.

- $\tilde{x} \leftarrow \mathrm{GC} \cdot \operatorname{inp}(x, s)$ : Gets an input $x$ and the same key $s$. Outputs the garbled input $\tilde{x}$.

- $y \leftarrow \operatorname{GC} \cdot \operatorname{eval}(\tilde{C}, \tilde{x}):$ Gets a garbled circuit $\tilde{C}$ and matching input $\tilde{x}$, and computes the output.

We require that for any circuit $C$, input $x$, setting $(\tilde{C}, s) \leftarrow \mathrm{GC} \cdot \operatorname{circ}\left(1^{\lambda}, C\right)$ and $\tilde{x} \leftarrow \mathrm{GC} \cdot \operatorname{inp}(x, s)$ we get $\mathrm{GC} \cdot \operatorname{eval}(\tilde{C}, \tilde{x})=C(x)$.

\subsection{Construction of Reusable GRAM}

Overview. We now show how to construct a reusable garbled-RAM scheme by combining reusable garbled circuit with a one-time garbled RAM scheme. An alternative construction of reusable garbled RAM from functional encryption for circuits appears in Appendix D, and it uses many of the same ideas shown here.

Let $\mathrm{GC}=$ (GC.circ, GC.inp, GC.eval) be a reusable garbled circuit scheme whose required security properties we specify later and GR1 = (GR1.prog, GR1.inp, GR1.eval) be a one-time garbled-RAM scheme. Recall our first approach from the introduction, which was to consider the circuit $C[P](r, x)$ that has $P$ hard-wired in and gets as input randomness $r$ and input $x$. The circuit $C[P](r, x)$ runs $\left(\tilde{P}_{\text {one }}, s\right) \leftarrow$ GR1.prog $\left(1^{\lambda}, P,(\cdots)\right)$ and $\tilde{x}_{\text {one }} \leftarrow \mathrm{GR} 1 . \operatorname{inp}(x, s,(\cdots))$, using $r$ as randomness, and outputs $\left(\tilde{P}_{\text {one }}, \tilde{x}_{\text {one }}\right)$. Our hope was to create a reusable garbled circuit $\tilde{C} \leftarrow \mathrm{GC} \cdot \operatorname{circ}(C[P])$ as our reusable garbled RAM program.

Observe that the circuit $C[P]$ from above has short input and very long output, related to the running time of $P$. Unfortunately, the construction of reusable garbled circuits of Goldwasser et al. $\left[\mathrm{GKP}^{+} 13 \mathrm{~b}\right]$, requires that the size of the garbled input to the circuit always exceeds the size of the circuit's output, even if the size of the actual input of the circuit is small. In particular, they have output-size dependence. In Appendix $\mathrm{C}$, we show that any reusable circuit garbling scheme with simulation-based security must have output-size dependence. In our context, that would mean that garbling an input to the program takes as much time as evaluating a program and therefore would not be useful in the context of outsourcing. 
We fix the problem above by tweaking the above construction in a way that allows us to reduce the security requirement on the reusable garbled circuit to something weaker than simulation security, while still achieving simulation security for our final construction. In particular, we first present our modified construction of reusable garbled RAM from reusable garbled circuits, then we present a new notion of security for reusable garbled circuits that we call "distributional indistinguishability", and show that is suffices to make our construction secure (Section 3.4) and finally, we show how to instantiate this new notion of reusable garbled circuits (Section 3.5) while achieving output-size independent efficiency.

The main modification that we make to the first-attempt construction from above is to first transform a program $P$ with run-time $t$ into a modified program $P^{+}$that we call the real-or-dummy program. In addition to the input $x$, the new program $P^{+}$takes also an alleged output $y$ and a flag $\psi$. If $\psi=1$ (real) then $P^{+}(x, \psi, y)$ simply executes $P(x)$, ignoring $y$. If If $\psi=0$ (dummy), on the other hand, then $P^{+}$simply executes $t$ dummy steps and outputs $y$, ignoring $x$.

Just as before, we consider the circuit $C\left[P^{+}\right](r,(x, \psi, y))$ that outputs a one-time garbled program $\tilde{P}$ garbling $P^{+}$and garbled input $\tilde{x}$ garbling $(x, \psi, y)$ using $r$ as randomness. We then construct a reusable garbled circuit $\tilde{C}$ garbling $C\left[P^{+}\right]$as the reusable garbled RAM program. Intuitively, the simulator of the reusable garbled RAM will simply provide a garbling of a "dummy input" consisting of $\left(r, 0^{n}, \psi=0, y\right)$ instead of the "real" input $\left(r, x, \psi=1,0^{m}\right)$. Proving security of the new construction boils down to proving that, given $\tilde{C}$, one cannot distinguish many garbling of real inputs vs. dummy inputs. Notice that the outputs $\tilde{P}, \tilde{x}$ derived from real-inputs vs. dummyinputs look indistinguishable by the security of the one-time garbled RAM. Therefore, we reduce simulation-based security of the full scheme to showing a new type of "distributional indistinguishability" for reusable garbled circuits, where it should be hard to distinguish garbled inputs from two different distributions (e.g., read or dummy) that produce indistinguishable outputs. This idea is similar in spirit to one used by De Caro et al. $\left[\mathrm{CIJ}^{+} 13\right]$ to convert indistinguishability-based security to simulation-based security for functional encryption. However, our notion of "distributional indistinguishability" is new.

The real-or-dummy program $\mathbf{P}^{+}$. In more detail, for a RAM program $P$ with input-size $n$, output-size $m$ and running-time bound $t$, let $P^{+}$be a RAM program that gets as input $(x, \psi, y)$ with $|x|=n,|\psi|=1$ and $|y|=m$. If $\psi=1$ then $P^{+}(x, \psi, y)$ outputs $P(x)$, and if $\psi=0$ then it outputs $y$ after $t$ steps. Note that the complexity of $P^{+}$is essentially the same as $P$, except that it has input of size $n+m+1$ rather than just $n$.

The program-garbling circuit. A central component of our construction is a circuit that runs the program- and input-garbling routines of the underlying one-time GRAM scheme. For a RAM program $P$ with input-size $n$, output-size $m$ and running-time bound $t$, and for security parameter $\lambda$, define $C[P, n, m, t, \lambda]$ as the following circuit with $n+m+2 \lambda+1$ input bits: ${ }^{7}$

$\mathbf{C}[\mathbf{P}, \mathbf{n}, \mathbf{m}, \mathbf{t}, \lambda](r, x, \psi, y): \quad / / r=\left(r_{1}, r_{2}\right) \in\{0,1\}^{2 \lambda}, x \in\{0,1\}^{n}, \psi \in\{0,1\}, y \in\{0,1\}^{m}$

1. Run $\left(\tilde{P}_{\text {one }}, s_{\text {one }}\right) \leftarrow \mathrm{GR} 1 . \operatorname{prog}\left(1^{\lambda}, P^{+},(n, m, t) ; r_{1}\right), \tilde{x}_{\text {one }} \leftarrow \mathrm{GR} 1 . \operatorname{inp}\left((x, \psi, y), s_{\text {one }},(n, m, t) ; r_{2}\right)$, 2. Output $\left(\tilde{P}_{\text {one }}, \tilde{x}_{\text {one }}\right)$.

GR: Reusable Garbled RAM Construction. We describe our reusable GRAM construction $\mathrm{GR}=$ (GR.prog, GR.inp, GR.eval) in the following figure.

\footnotetext{
${ }^{7}$ For simplicity, we assume that GR1.prog, GR1.inp uses exactly $\lambda$ bits of randomness each. This can always be made the case by using a pseudorandom generator.
} 
$(\tilde{P}, s) \leftarrow \mathrm{GR} \cdot \operatorname{prog}\left(1^{\lambda}, P,(n, m, t)\right)$ :

1. Construct the circuit $C[P, n, m, t, \lambda]$ as shown above.

2. Compute a garbling of this circuit $(\tilde{C}, s) \leftarrow \mathrm{GC} \cdot \operatorname{circ}\left(1^{\lambda}, C[P, n, m, t, \lambda]\right)$, and output $\tilde{P}:=\tilde{C}, s$.

$\tilde{x} \leftarrow \operatorname{GR} \cdot \operatorname{inp}(x, s,(n, m, t))$ :

1. Choose a random $r \leftarrow\{0,1\}^{2 \lambda}$, set $\psi=1, y=0^{m}$, and $w=(r, x, \psi, y)$,

2. Garble the input to $C[P \ldots]$, outputting $\tilde{w} \leftarrow \mathrm{GC} \cdot \operatorname{inp}(w, s)$.

$y=\operatorname{GR} \cdot \operatorname{eval}(\tilde{P}, \tilde{w}):$

1. Evaluate the garbled circuit $\tilde{C}:=\tilde{P}$ to get $\left(\tilde{P}_{\text {one }}, \tilde{x}_{\text {one }}\right) \leftarrow \mathrm{GC}$.eval $(\tilde{C}, \tilde{w})$.

2. Evaluate the 1-time GRAM and output $y=\operatorname{GR} 1$.eval $\left(\tilde{P}_{\text {one }}, \tilde{x}_{\text {one }}\right)$.

\subsection{Simulation Security From Distributional Indistinguishability}

A crucial observation is that we can prove simulation-security for the above reusable GRAM construction GR using a new notion of "distributional indistinguishability" security for the underlying garbled circuit scheme and the usual simulation security for the underlying one-time GRAM scheme. "Distributional indistinguishability' says that one cannot distinguish garbled inputs from any two sets of independent distributions that produce individually indistinguishable outputs.

Definition 3.4. Let $\mathrm{GC}=$ (GC.circ, GC.inp, GC.eval) be a garbled circuit scheme. We say that GC provides distributional indistinguishability if for every circuit ensemble $C=\left\{C_{\lambda}\right\}$, every polynomial $q=q(\lambda)$, and every $2 q$ polynomial-time samplable distributions $D_{1}, \ldots, D_{q}$ and $D_{1}^{\prime}, \ldots, D_{q}^{\prime}$, if for all $j \in[q]$ it holds that $C\left(w_{j}\right) \stackrel{\text { comp }}{\approx} C\left(w_{j}^{\prime}\right)$ where $w_{j} \leftarrow D_{j}\left(1^{\lambda}\right), w_{j}^{\prime} \leftarrow D_{j}^{\prime}\left(1^{\lambda}\right)$ then it also holds that

$$
\left\langle\tilde{C}, \tilde{w}_{1}, \ldots \tilde{w}_{q}\right\rangle \stackrel{\text { comp }}{\approx}\left\langle\tilde{C}, \tilde{w}_{1}^{\prime}, \ldots \tilde{w}_{q}^{\prime}\right\rangle
$$

where $(\tilde{C}, s) \leftarrow \mathrm{GC} \cdot \operatorname{circ}\left(1^{\lambda}, C_{\lambda}\right), w_{i} \leftarrow D_{i}\left(1^{\lambda}\right), w_{i}^{\prime} \leftarrow D_{i}^{\prime}\left(1^{\lambda}\right), \tilde{w}_{i} \leftarrow \mathrm{GC} \cdot \operatorname{inp}\left(w_{i}, s\right), \tilde{w}_{i}^{\prime} \leftarrow \mathrm{GC} \cdot \operatorname{inp}\left(w_{i}^{\prime}, s\right)$.

We say that the scheme has security with public input garbling if the above holds when we include the garbling key s in the two distributions on the bottom.

We remark that this notion is clearly implied by simulation security for reusable garbled circuits, but simulation security of reusable garbled circuits requires "output-size dependence" where the size of the garbled input must exceed that of the circuit's output (see Appendix C). Furthermore, we remark that for any scheme with public input garbling, distributional indistinguishability for $q=1$ implies security for arbitrary $q$ by a simple hybrid argument. Interestingly, this does not hold for simulation security where simulation security for $q=1$ does not seem to imply security for a larger $q$, even if the scheme has public input garbling.

In Section 3.5 we show how to construct a reusable circuit-garbling scheme with output-size independence satisfying this definition using indistinguishability obfuscation.

Theorem 3.5. If $\mathrm{GC}=(\mathrm{GC} . c i r c, \mathrm{GC} . \mathrm{inp}, \mathrm{GC}$. eval) is a reusable garbled-circuit scheme satisfying distributional indistinguishability and GR1 = (GR1.prog, GR1.inp, GR1.eval) is a one-time garbledRAM scheme, then the scheme GR from above is a reusable garbled-RAM scheme satisfying simulation security. Furthermore, if GC has security with public input garbling, then so does GR.

See Appendix F.1 for a formal proof of the above theorem. On an intuitive level, the simulator for the GR scheme will simply rely on the "dummy mode" of the program $P^{+}$by garbling inputs to the reusable garbled circuit that have the flag $\psi=0$ (indicating dummy mode) and a hard-coded output $y_{i}$. Such inputs result in outputs of the reusable garbled circuit that are distributionally indistinguishable from real, by the security of the 1-time garbled RAM GR1. 


\subsection{Achieving Distributional Indistinguishability}

We now construct reusable garbled circuits with "distributional indistinguishability" and "outputsize independent efficiency". Furthermore, our construction has public input-garbling. The construction is based on "indistinguishability obfuscation" (see Appendix A.1) and a NIZK which is "statistically simulation sound" (see Appendix A.2). It is inspired by the construction of functional encryption from indistinguishability obfuscation of $\left[\mathrm{GGH}^{+} 13 \mathrm{~b}\right]$.

Construction. Let $\mathcal{O}$ be an obfuscation scheme, let $\mathcal{P} \mathcal{K} \mathcal{E}=$ (Setup, Encrypt, Decrypt) be a public key encryption scheme, and let $\Pi=(K, P, V)$ be a NIZK scheme with statistical simulation soundness. Let $L_{E Q}$ be the NP language defined as

$$
L_{E Q}=\left\{\left(\mathrm{pk}_{1}, \mathrm{pk}_{2}, c_{1}, c_{2}\right): \exists m, r_{1}, r_{2}, \quad c_{1}=\operatorname{Encrypt}\left(\mathrm{pk}_{1}, m ; r_{1}\right) \wedge c_{2}=\operatorname{Encrypt}\left(\mathrm{pk}_{2}, m ; r_{2}\right)\right\}
$$

For any circuit $C:\{0,1\}^{n} \rightarrow\{0,1\}^{m}$ define the circuit $C^{*}\left[\sigma, \mathrm{pk}_{1}, \mathrm{pk}_{2}, b, \mathrm{sk}, u, v\right]$ where: $\sigma$ is a CRS for the NIZK, $\mathrm{pk}_{1}, \mathrm{pk}_{2}$ are encryption keys, $b \in\{1,2\}$ is an index, sk is the decryption key for $\mathrm{pk}_{b}, u$ is of size $\left|\left(c_{1}, c_{2}, \pi\right)\right|$ where $c_{1}, c_{2}$ are ciphertexts of $n$-bit messages and $\pi$ is a NIZK for $L_{E Q}$, and $v \in\{0,1\}^{m}$.

$C^{*}\left[\sigma, \mathrm{pk}_{1}, \mathrm{pk}_{2}, b, \mathrm{sk}, u, v\right]\left(c_{1}, c_{2}, \pi\right):$

1. If $u=\left(c_{1}, c_{2}, \pi\right)$ output $v$.

2. Verify that $\pi$ is a proof of $\left(\mathrm{pk}_{1}, \mathrm{pk}_{2}, c_{1}, c_{2}\right) \in L_{E Q}$ by running $V\left(\sigma,\left(\mathrm{pk}_{1}, \mathrm{pk}_{2}, c_{1}, c_{2}\right), \pi\right)$. If this rejects, output $\perp$.

3. Compute $x=\operatorname{Decrypt}\left(\right.$ sk, $\left.c_{b}\right)$. Output $C(x)$.

We define the circuit garbling scheme GC = (GC.circ, GC.inp, GC.eval), which has public input garbling, as follows:

- $(\tilde{C}, s) \leftarrow \mathrm{GC} \cdot \operatorname{circ}\left(1^{\lambda}, C\right):$ Generate $\left(\mathrm{pk}_{1}, \mathrm{sk}_{1}\right) \leftarrow \operatorname{Setup}\left(1^{\lambda}\right),\left(\mathrm{pk}_{2}, \mathrm{sk}_{2}\right) \leftarrow \operatorname{Setup}\left(1^{\lambda}\right), \sigma \leftarrow$ $K\left(1^{\lambda}\right)$. Construct the circuit $C^{*}:=C^{*}\left[\sigma, \mathrm{pk}_{1}, \mathrm{pk}_{2}, 1, \mathrm{sk}_{1}, u=\perp, v=\perp\right]$ from $C$ as shown. Output $\tilde{C} \leftarrow \mathcal{O}\left(1^{\lambda}, C^{*}\right)$ and $s:=\left(\sigma, \mathrm{pk}_{1}, \mathrm{pk}_{2}\right)$.

- $\tilde{x} \leftarrow \mathrm{GC} \cdot \operatorname{inp}(x, s)$ : output $\tilde{x}:=\left(c_{1}, c_{2}, \pi\right)$, where $c_{1} \leftarrow \operatorname{Encrypt}\left(\mathrm{pk}_{1}, x ; r_{1}\right), c_{2} \leftarrow \operatorname{Encrypt}\left(\mathrm{pk}_{2}, x ; r_{2}\right)$ and $\pi \leftarrow P\left(\sigma,\left(\mathrm{pk}_{1}, \mathrm{pk}_{2}, c_{1}, c_{2}\right),\left(r_{1}, r_{2}\right)\right)$ is an NIZK that $\left(\mathrm{pk}_{1}, \mathrm{pk}_{2}, c_{1}, c_{2}\right) \in L_{E Q}$.

- GC.eval $(\tilde{C}, \tilde{x})$ : Interpret $\tilde{C}$ as an obfuscated circuit and output $\tilde{C}(\tilde{x})$.

See Appendix F.2 for a proof of the following theorem.

Theorem 3.6. If $\mathcal{O}$ is an indistinguishability obfuscator, $\Pi$ is a statistical-simulation-sound (SSS) $N I Z K$, and $\mathcal{P} \mathcal{K} \mathcal{E}$ is a semantically secure encryption scheme, then the above construction GC is a reusable garbled circuit with distributional indistinguishability and public input garbling.

\section{Reusable Garbled RAM with Persistent Memory}

We now move to consider the harder setting with persistent memory. The construction is very similar to the one above, in particular here too we construct a reusable garbled-RAM scheme by combining a reusable garbled circuit with a one-time garbled RAM scheme. The main differences are that the one-time GRAM that we use has persistent memory, and the garbled circuit satisfies a stronger notion of security. 


\subsection{Definitions}

A GRAM scheme with persistent memory has an additional procedure GR.data used to garbled the initial memory data $D$ to a garbled data $\tilde{D}$. We envision the case where the user has a program $P$ and wants to run many executions of this program with different "short-inputs" $x_{i}$ where each execution $P\left(x_{i}\right)$ can read and write to the persistent memory, and the changes persist for future executions. This means that the order of executions is important and does not commute. In particular, we need to ensure that the server only learns the outputs of the executions when performed in the correct order and cannot (e.g.,) reorder the executions or roll-back the changes made by one execution when performing the next execution.

In order to do this, we need to incorporate the index $i$ of the execution into the program/input garbling procedures. In the case of reusable schemes the user garbled the program only once to get the garbled version $\tilde{P}$, and then can garble many different inputs $\tilde{x}_{i}$, so in this case only the input garbling is given the index $i$. For one-time scheme the user has to garble the program $P$ afresh for each new execution to get a garbled program $\tilde{P}_{i}$ and garbled input $\tilde{x}_{i}$, so in this case both the program and input garbling procedures are given the index $i$.

Definition 4.1 (GRAM with persistent memory). A garbled RAM (GRAM) scheme with persistent memory consists of four procedures: GR =(GR.data, GR.prog, GR.inp, GR.eval):

- $(\tilde{D}, k) \leftarrow \operatorname{GR} \cdot d a t a\left(1^{\lambda}, D\right):$ Gets data $D \in\{0,1\}^{N}$ and outputs the garbled data $\tilde{D}$ and a data-key $k$.

- $(\tilde{P}, s) \leftarrow \mathrm{GR} \cdot \operatorname{prog}(P, k,(N, n, m, t),[i]):$ Gets a RAM program $P$, data-key $k$, and bounds on the memory size $N$, input size $n$, output size $m$, and run-time $t$ (say that all bounds encoded in binary). For one-time scheme, the procedure is also given an index $i$ indicating the order in which this program is to be executed. Outputs a garbled program $\tilde{P}$ and program-key s.

- $\tilde{x} \leftarrow \mathrm{GR} \cdot \operatorname{inp}(x, k, s,(N, n, m, t), i)$ : Takes as input an $n$-bit value $x$, the keys $k, s$, and an index $i$ for the order of the execution. It outputs the garbled input $\tilde{x}$.

- $y=\mathrm{GR}_{\operatorname{eval}}{ }^{\tilde{D}}(\tilde{P}, \tilde{x})$ : This is a RAM program that takes $(\tilde{P}, \tilde{x})$ as input, has memory $\tilde{D}$, and computes the output $y$. The program updates the memory contents of $\tilde{D}$ during its execution and these changes persist for the following execution.

Correctness. Consider initial memory data $D \in\{0,1\}^{N}$, program $P$ with bounds $(N, n, m, t)$ and a sequence of inputs $x_{i} \in\{0,1\}^{n}$ for $i=1, \ldots, q$. Let $y_{i}=P^{D}\left(x_{i}\right)$ where the executions are performed in the correct order starting with $i=1$ and the memory data $D$ is updated with each execution. We define one-time and reusable correctness separately.

One-time: Consider running $(\tilde{D}, k) \leftarrow \mathrm{GR} \cdot \operatorname{data}\left(1^{\lambda}, D\right),\left(\tilde{P}_{i}, s_{i}\right) \leftarrow \mathrm{GR} \cdot \operatorname{prog}(P, k,(N, n, m, t), i)$, $\tilde{x}_{i} \leftarrow \mathrm{GC} . \operatorname{inp}\left(x_{i}, k, s_{i}\right), y_{i}^{\prime} \leftarrow \mathrm{GC}_{\text {eval }}{ }^{\tilde{D}}\left(\tilde{P}_{i}, \tilde{x}_{i}\right)$ where the evaluations are executed in the correct order and the garbled memory $\tilde{D}$ is updated with each evaluation. We require that $y_{i}^{\prime}=y_{i}$ for all $i \in[q]$ with probability 1.

Reusable: Consider running $(\tilde{D}, k) \leftarrow \mathrm{GR} \cdot \operatorname{data}\left(1^{\lambda}, D\right),(\tilde{P}, s) \leftarrow \mathrm{GR} \cdot \operatorname{prog}(P, k,(N, n, m, t)), \tilde{x}_{i} \leftarrow$ $\mathrm{GC} . \operatorname{inp}\left(x_{i}, k, s, i\right), y_{i}^{\prime} \leftarrow \mathrm{GC} \cdot \mathrm{eval}^{\tilde{D}}\left(\tilde{P}, \tilde{x}_{i}\right)$ where the evaluations are executed in the correct order and the garbled memory $\tilde{D}$ is updated with each evaluation. We require that $y_{i}^{\prime}=y_{i}$ for all $i \in[q]$ with probability 1.

Efficiency. We will analyze the efficiency of our constructions in Section 5. On a high level, we will always require that the data-garbling GR.data runs in time $N \cdot \operatorname{poly}(\lambda)$, the run-time of 
GR.prog and GR.eval should only scale linearly with $t$ and poly-logarithmically with $N$, and and the run-time of GR.inp should only depend logarithmically on $t, N$. We will also discuss compact solutions where the run-time of GR.prog is only logarithmic in $t$.

Security. We require the usual simulation-based security from our garbled-RAM constructions. To simplify notation, we define a polynomial size input specification in $=\left((N, n, m, t), D, P,\left\langle x_{i}\right\rangle_{i \in[q]}\right)$ as consisting of polynomial bounds $(N, n, m, t)$, initial memory data $D \in\{0,1\}^{N}$, program $P$, and a polynomial-size sequence of inputs $x_{i} \in\{0,1\}^{n}$ for $i=1, \ldots, q$. We define the corresponding output specification out $=\left((N, n, m, t), P,\left\langle y_{i}\right\rangle_{i \in[q]}\right)$ with $y_{i}=P^{D}\left(x_{i}\right)$ where the executions are performed in the correct order starting with $i=1$ and the memory data $D$ is updated with each execution. Intuitively, the output specification is the only thing that the evaluator should learn from the garbled data/program/input.

Definition 4.2 (GRAM with Persistent Memory ). Let GR = (GR.data, GR.prog, GR.inp, GR.eval) be a garbled RAMs with persistent memory. We say GR has one-time/reusable security, if there exists a simulator $\operatorname{Sim}$ such that for any poly-size input specification in $=\left((N, n, m, t), D, P,\left\langle x_{i}\right\rangle_{i \in[q]}\right)$ with corresponding output specification out $=\left((N, n, m, t), P,\left\langle y_{i}\right\rangle_{i \in[q]}\right)$ we have

$$
\operatorname{Real}[\text { in, } \lambda] \stackrel{\operatorname{comp}}{\approx} \operatorname{Sim}\left(1^{\lambda}, \text { out }\right)
$$

where Real[in, $\lambda]$ is defined separately for one-time and reusable security as follows:

One-Time. Define Real $[$ in, $\lambda]=\left(\tilde{D},\left\langle\tilde{P}_{i}, \tilde{x}_{i}\right\rangle_{i \in[q]}\right)$ where $(\tilde{D}, k) \leftarrow \operatorname{GR} \cdot \operatorname{data}\left(1^{\lambda}, D\right)$, and for $i \in[q]$ $\left(\tilde{P}_{i}, s_{i}\right) \leftarrow \mathrm{GR} \cdot \operatorname{prog}(P, k,(N, n, m, t), i), \tilde{x}_{i} \leftarrow \mathrm{GC} \cdot \operatorname{inp}\left(x_{i}, k, s_{i}\right)$.

Reusable. Define Real $[$ in, $\lambda]=\left(\tilde{D}, \tilde{P},\left\langle\tilde{x}_{i}\right\rangle_{i \in[q]}\right)$ where $(\tilde{D}, k) \leftarrow \operatorname{GR} \cdot \operatorname{data}\left(1^{\lambda}, D\right)$, $(\tilde{P}, s) \leftarrow \mathrm{GR} \cdot \operatorname{prog}(P, k,(N, n, m, t))$, and for $i \in[q]: \tilde{x}_{i} \leftarrow \mathrm{GC} \cdot \operatorname{inp}\left(x_{i}, k, s, i\right)$.

We require that $\operatorname{Sim}$ runs in time poly $(N, t, n, m, q, \lambda)$.

Remarks on Definition. For simplicity, we only consider the scenario involving a single program $P$. This is without loss of generality as $P$ can be a universal $R A M$ that executes code stored in memory at a location which is indicated as part of the short input $x$. This approach of storing code in memory most closely resembles computation in real life. We also do not explicitly consider program privacy and assume that the code of the program is public. (The approach of setting $P$ to be a universal RAM and executing programs stored in memory also provides privacy for the code of the programs being executed, see Section 6.)

Note that garbled RAM with persistent memory cannot have public input garbling, as this allows the attacker to learn more information than allowed about the garbled database $D$ by executing its own programs over it. Hence, the data key $k$ is kept secret and the evaluator only learns the outputs of the specified program $P$ with specified inputs $x_{i}$ when executed in the correct order. This prevents the attacker from, e.g., learn the outputs of additional programs, roll back the changes to data made by one program and see how it affects the outputs of future programs, etc.

The works of [LO13a, $\left.\mathrm{GHL}^{+} 14\right]$ provide constructions of one-time GRAM with persistent memory satisfying the above definition. The syntax of $\left[\mathrm{GHL}^{+} 14\right]$ is somewhat more complicated since it considers a scenario with many different programs $P_{i}$, but it is easy to see that it implies our simplified syntax/definition. Seer Appendix B for an overview of the construction. 


\subsection{Constructing Reusable GRAM with Persistent Memory}

The construction of garbled RAM scheme with persistent memory from a one-time garbled RAM and a reusable garbled circuits is very similar to the case without persistent memory, and we essentially just make the needed syntactic changes to accommodate the persistent memory. However, the security analysis will require some more substantial changes.

Let $G C=$ (GC.circ, GC.inp, GC.eval) be a reusable garbled circuit scheme and GR1 = (GR1.data, GR1.prog, GR1.inp, GR1.eval) be a one-time garbled-RAM scheme with persistent memory. For a program $P$, let $P^{+}$be the "real-or-dummy" program defined previously. For a RAM program $P$, with memory-size $N$, input-size $n$, output-size $m$, running-time bound $t$, and security parameter $\lambda$, define $C[P, N, n, m, t, \lambda]$ as the following circuit with $n+m+2 \lambda+1$ input bits:

\begin{tabular}{|c|c|}
\hline$\underline{\mathbf{C}[\mathbf{P}, \mathbf{N}, \mathbf{n}, \mathbf{m}, \mathbf{t}, \lambda]\left(k, r=\left(r_{1}, r_{2}\right), x, \psi, y, i\right)}:$ & $\begin{array}{l}/ / k, r_{1}, r_{2} \in\{0,1\}^{\lambda} \\
/ / x \in\{0,1\}^{n}, \psi \in\{0,1\}, y \in\{0,1\}^{m}\end{array}$ \\
\hline $\begin{array}{l}\text { 1. Run }\left(\tilde{P}_{\text {one }}, s_{\text {one }}\right) \leftarrow \mathrm{GR} 1 \cdot \operatorname{prog}\left(P^{+}, k,(N, m\right. \\
\tilde{x}_{\text {one }} \leftarrow \mathrm{GR} 1 . \operatorname{inp}\left((x, \psi, y), k, s_{\text {one }} ; r_{2}\right), \\
\text { 2. Output }\left(\tilde{P}_{\text {one }}, \tilde{x}_{\text {one }}\right) .\end{array}$ & $\left.+n+1, m, t), i ; r_{1}\right)$ \\
\hline
\end{tabular}

Reusable Garbled RAM with Persistent Memory. We describe the scheme GR in the following figure.

$(\tilde{D}, k) \leftarrow \mathrm{GR} . \operatorname{data}\left(1^{\lambda}, D\right)$ : Use the the underlying GR1 and output: $(\tilde{D}, k) \leftarrow \mathrm{GR} 1 . \operatorname{data}\left(1^{\lambda}, D\right)$.

$(\tilde{P}, s) \leftarrow \mathrm{GR} \cdot \operatorname{prog}(P, k,(N, n, m, t))$ :

1. Construct the circuit $C=C[P, N, n, m, t, \lambda]$,

2. Garble this circuit $(\tilde{C}, s) \leftarrow \mathrm{GC} \cdot \operatorname{circ}\left(1^{\lambda}, C\right)$ and output $\tilde{P}=\tilde{C}, s$.

$\tilde{w} \leftarrow \operatorname{GR} \cdot \operatorname{inp}(x, k, s, i):$

1. Choose a random $r \leftarrow\{0,1\}^{2 \lambda}$, set $\psi=1, y=0^{m}$, and $w=(k, r, x, \psi, y, i)$,

2. Garble the input to $C[P \ldots]$, outputting $\tilde{w} \leftarrow \mathrm{GC} \cdot \operatorname{inp}(w, s)$.

$y=\operatorname{GR} \cdot \operatorname{eval}(\tilde{P}, \tilde{w}):$

1. Set $\tilde{C}=\tilde{P}$ and evaluate the garbled circuit, $\left(\tilde{P}_{\text {one }}, \tilde{x}_{\text {one }}\right) \leftarrow \operatorname{GC} . \operatorname{eval}(\tilde{C}, \tilde{w})$.

2. Evaluate the 1-time GRAM and output $y \leftarrow \operatorname{GR} 1$.eval $\left(\tilde{P}_{\text {one }}, \tilde{x}_{\text {one }}\right)$.

Security. The proof of security is very similar to the case of no persistent memory, except that we need a stronger variant of "distributional indistinguishability". In the previous case without persistent memory, each garbled input $w_{i}=\left(r_{i}, x_{i}, \psi=1,0^{m}\right)$ was chosen with its own fresh and independent randomness $r_{i}$ and there was no relationship between different garbled inputs. In the case of persistent memory, the inputs $w_{i}=\left(k, r_{i}, x_{i}, \psi=1,0^{m}, i\right)$ all include the same "data key" $k$. In other words, the inputs have some common correlated secret and we cannot argue that they come from independent distributions $D_{i}, D_{i}^{\prime}$. Therefore, we are forced to rely on a stronger notion of security of reusable garbled circuit than Definition 3.4, a notion we define below.

Definition 4.3 (Correlated Distributional Indistinguishability). Let GC = (GC.circ, GC.inp, GC.eval) be a garbled circuit scheme. We say that GC provides correlated distributional indistinguishability if for every circuit ensemble $C=\left\{C_{\lambda}\right\}$ and two PPT distribution samplers $D, D^{\prime}$ over vectors of inputs to $C$, if the two distributions satisfy

$$
\left\langle C_{\lambda}\left(w_{1}\right), \ldots, C_{\lambda}\left(w_{q}\right), \text { aux }\right\rangle \stackrel{\mathrm{comp}}{\approx}\left\langle C_{\lambda}\left(w_{1}^{\prime}\right), \ldots, C_{\lambda}\left(w_{q}^{\prime}\right), \text { aux } x^{\prime}\right\rangle
$$


where $\left(w_{1}, \ldots, w_{q}\right.$, aux $) \leftarrow D\left(1^{\lambda}\right),\left(w_{1}^{\prime}, \ldots, w_{q}^{\prime}\right.$, aux $) \leftarrow D^{\prime}\left(1^{\lambda}\right)$, then also

$$
\left\langle\tilde{C}, \tilde{w}_{1}, \ldots \tilde{w}_{q}, \text { aux }\right\rangle \stackrel{\text { comp }}{\approx}\left\langle\tilde{C}, \tilde{w}_{1}^{\prime}, \ldots \tilde{w}_{q}^{\prime}, \text { aux }\right\rangle
$$

where $(\tilde{C}, s) \leftarrow \mathrm{GC} \cdot \operatorname{circ}\left(1^{\lambda}, C_{\lambda}\right)$ and $\tilde{w}_{i} \leftarrow \mathrm{GC} \cdot \operatorname{inp}\left(w_{i}, s\right), \tilde{w}_{i}^{\prime} \leftarrow \mathrm{GC} \cdot \operatorname{inp}\left(w_{i}^{\prime}, s\right)$.

We note that this definition is still weaker than simulation security, but stronger than Definition 3.4 (which considers only $w_{i}$ 's that are sampled independently). The proof of the following theorem is similar to that of Theorem 3.5.

Theorem 4.4. If $\mathrm{GC}=$ (GC.circ, GC.inp, GC.eval) is a reusable garbled-circuit scheme with outputsize independence satisfying correlated distributional indistinguishability and GR1 = (GR1.data, GR1.prog, GR1.inp, GR1.eval) is a garbled-RAM scheme with persistent memory satisfying one-time simulation security, then the scheme GR from above is a reusable garbled-RAM scheme with persistent memory satisfying simulation security (Definition 4.2).

See Appendix F.3 for a proof of the above theorem, which closely follows the proof of Theorem 3.5.

\subsection{Achieving Correlated Distributional Indistinguishability}

Below we present two candidate constructions of reusable garbled circuits from obfuscation. The first one is very simple, but essentially relies on a "special-purpose" virtual black-box (VBB) obfuscation conjecture of some relevant functionality. The second construction is slightly more complex, and we can prove its security under an notion of obfuscation that we call strong differing-inputs obfuscation (and which generalizes differing-inputs obfuscation $\left[\mathrm{BGI}^{+} 12, \mathrm{BCP} 13, \mathrm{ABG}^{+} 13\right]$ ). Technically, the two assumptions needed to prove security of our two constructions are incomparable, and therefore we present both options.

Construction 1 (Obfuscating a "decrypt-then-evaluate" circuit). Let $\mathcal{P} \mathcal{K} \mathcal{E}=$ (Setup, Encrypt, Decrypt) be a public-key encryption scheme. For any circuit $C:\{0,1\}^{n} \rightarrow\{0,1\}^{m}$ and decryption secret key sk we define the circuit $C^{*}[\mathrm{sk}](c)$ which computes $x=\operatorname{Decrypt}(\mathrm{sk}, c)$ and outputs $C(x)$. We define the circuit garbling scheme $\mathrm{GC}=(\mathrm{GC}$.circ, GC.inp, GC.eval) as follows:

- GC. $\operatorname{circ}\left(1^{\lambda}, C\right)$ : Generate $(\mathrm{pk}, \mathrm{sk}) \leftarrow \operatorname{Setup}\left(1^{\lambda}\right)$. Construct the circuit $C^{*}:=C^{*}[\mathrm{sk}]$ from $C$ as shown. Output $\tilde{C} \leftarrow \mathcal{O}\left(1^{\lambda}, C^{*}\right)$ and $s:=$ pk.

- GC.inp $(x, s)$ : Output $\tilde{x} \leftarrow \operatorname{Encrypt}(\mathrm{pk}, x)$.

- GC.eval $(\tilde{C}, \tilde{x})$ : Interpret $\tilde{C}$ as an obfuscated circuit and output $\tilde{C}(\tilde{x})$.

We simply conjecture that this construction is secure when instantiated with a standard-construction PKE and a "good" obfuscator, such as the candidate construction of [GGH+13b].

Conjecture 4.5. There exists a CCA-secure public-key encryption scheme $\mathcal{P} \mathcal{K} \mathcal{E}$ and an obfuscator $\mathcal{O}$, for which the above construction GC is a reusable garbled circuit with correlated distributional indistinguishability.

As a "sanity check", it's easy to show that the conjecture holds if the attacker were only given black-box access to the circuit $C^{*}[\mathrm{sk}]$ rather than the obfuscated circuit $\tilde{C} \leftarrow \mathcal{O}\left(1^{\lambda}, C^{*}[\mathrm{sk}]\right)$. In particular, this follows from a sequence of hybrids where: (I) one-by-one, we modify the $i$ th garbled input $\tilde{w}_{i}$ to be an encryption of 0 instead of $w_{i}$ but make $C^{*}[\mathrm{sk}]$ return $w_{i}$ when queried with $\tilde{w}_{i}$ 
(CCA-security), (II) we modify all the values returned by $C^{*}[\mathrm{sk}]$ from $w_{i}$ to $w_{i}^{\prime}$ and switch aux to aux' (correlated ind. assumption) and (III) we go back one-by-one and modify $i$ th garbled input to be an encryption of $w_{i}^{\prime}$ and $C^{*}[\mathrm{sk}]$ to decrypt correctly (CCA-security). We note that our conjecture does not formally require full VBB security and, for example, is not defined using a simulator. None of the known negative results for obfuscation seems to apply to the above conjecture, when instantiated with a "natural" public-key encryption (e.g., Cramer-Shoup construction [CS98]).

Construction 2 (Using Strong Differing-Inputs Obfuscators). Next we present a construction for reusable garbled circuits with correlated distributional indistinguishability, which we prove secure under a new stronger form of the differing-inputs obfuscation assumption. Differing-inputs obfuscation (diO) $\left[\mathrm{BGI}^{+} 12, \mathrm{BCP} 13, \mathrm{ABG}^{+} 13\right]$ guarantees that for any two circuits $C_{0}$ and $C_{1}$, if it is difficult to find an input $x$ on which $C_{0}(x) \neq C_{1}(x)$, then it should be hard to distinguish the obfuscation of $C_{0}$ from the obfuscation of $C_{1}$. We define a stronger version of the assumption which maintains the indistinguishability of the obfuscations even when given several points $x_{1}, \ldots, x_{n}$ on which the two circuits have different but computationally indistinguishable outputs, as long as it is hard to find any other inputs on which the circuits differ. In particular, feeding these inputs $x_{i}$ to the obfuscated circuit does not help distinguish $C_{0}$ from $C_{1}$. We formalize this intuition in the following definition.

Definition 4.6. A family of circuits $\mathcal{C}$ with a sampler $\left(C_{0}, C_{1}, x_{1}, \ldots, x_{n}\right.$, aux $x_{0}$, aux 1$) \leftarrow \operatorname{Sam}\left(1^{\lambda}\right)$, which samples $C_{0}, C_{1} \in \mathcal{C}$ together with inputs $x_{1}, \ldots, x_{n}$ is said to be a strong differing inputs family if

$$
\left\{x_{1}, \ldots, x_{n}, C_{0}\left(x_{1}\right), \ldots, C_{0}\left(x_{n}\right), \text { aux }_{0}\right\} \stackrel{\text { comp }}{\approx}\left\{x_{1}, \ldots, x_{n}, C_{1}\left(x_{1}\right), \ldots, C_{1}\left(x_{n}\right), \text { aux } 1\right\}
$$

and for all PPT adversaries $\mathcal{A}$ there is a negligible function $\varepsilon$ such that

$$
\operatorname{Pr}\left[\begin{array}{c|c}
C_{0}(x) \neq C_{1}(x) & \left(C_{0}, C_{1}, x_{1}, \ldots, x_{n}, \operatorname{aux}_{0}, \operatorname{aux}_{1}\right) \leftarrow \operatorname{Sam}\left(1^{\lambda}\right) \\
\wedge x \notin\left\{x_{1}, \ldots, x_{n}\right\} & x \leftarrow \mathcal{A}\left(1^{\lambda}, C_{0}, C_{1}, x_{1}, \ldots, x_{n}, \operatorname{aux}_{0}, \operatorname{aux}_{1}\right)
\end{array}\right] \leq \varepsilon(\lambda) .
$$

Definition 4.7 (Strong Differing-Inputs Obfuscator (sdiO)). A PPT algorithm $\mathcal{O}$ satisfying correctness is a strong differing-inputs obfuscator (sdiO) for a strong differing-inputs family $\mathcal{C}$ with a sampler Sam, if for all PPT distinguisher algorithms $\mathcal{D}$, there is a negligible function $\varepsilon$ such that

$$
\left|\operatorname{Pr}\left[\mathcal{D}\left(1^{\lambda}, \mathcal{O}\left(1^{\lambda}, C_{0}\right), x_{1}, \ldots, x_{n}, \operatorname{aux}_{0}\right)=1\right]-\operatorname{Pr}\left[\mathcal{D}\left(1^{\lambda}, \mathcal{O}\left(1^{\lambda}, C_{1}\right), x_{1}, \ldots, x_{n}, \operatorname{aux}_{1}\right)=1\right]\right| \leq \varepsilon(\lambda),
$$

where $\left(C_{0}, C_{1}, x_{1}, \ldots, x_{n}\right.$, aux 0, aux $\left._{1}\right) \leftarrow \operatorname{Sam}\left(1^{\lambda}\right)$.

We note that this is a very strong definition, and it is not clear if it is preferable to Conjecture 4.5. The main advantage is that it is a general definition and not specifically tied to a particular construction. The recent work of [GGHW13] gives some evidence that general-purpose diO relative to arbitrary auxiliary input is unlikely to exist. The same negative result would hold for the stronger notion of general-purpose sdiO. However, the counterexample relies on highly contrived auxiliary input which is itself an obfuscation. Therefore, it still makes sense to assume diO and sdiO holds when the auxiliary input aux , aux $_{1}$ and the samples $x_{i}$ have some concrete structure. In our case, the auxiliary input and the points $x_{1}, \ldots, x_{n}$ will simply contain a public-key of an encryption scheme and some simulated NIZK proofs. Therefore, the results of [GGHW13] do not apply to sdiO for the restricted type of strong differing input circuit families $(\mathcal{C}$,Sam) that we rely on.

We also note that, for a differing inputs family for which the outputs of $C_{0}, C_{1}$ differ on the inputs $x_{1}, \ldots, x_{n}$, the circuits $C_{0}, C_{1}$ must be chosen from some high entropy distribution and 
cannot be known even given $\operatorname{aux}_{0}$, aux $x_{1}$. Otherwise, an attacker could simply feed the inputs to the two circuits and distinguish $C_{0}\left(x_{i}\right)$ from $C_{1}\left(x_{i}\right)$.

The construction of reusable garbled circuits from sdiO is similar to (but simpler than) the one from Section 3.4. Let $\mathcal{O}$ be a strong differing-inputs obfuscator, $\mathcal{P} \mathcal{K} \mathcal{E}=$ (Setup, Encrypt, Decrypt) be a semantically secure encryption scheme, and $\Pi=(K, P, V)$ be a simulation-sound NIZK scheme. For any circuit $C:\{0,1\}^{n} \rightarrow\{0,1\}^{m}$ we define a circuit $C^{*}\left[\sigma, \mathrm{pk}_{0}, \mathrm{pk}_{1}, b, \mathrm{sk}\right]\left(c_{0}, c_{1}, \pi\right)$, which takes as input two ciphertexts $c_{0}, c_{1}$ and an NIZK proof $\pi$ for $\left(\mathrm{pk}_{1}, \mathrm{pk}_{2}, c_{1}, c_{2}\right) \in L_{E Q}$, where $L_{E Q}$ is defined as in the previous section, and computes the following:

$$
\begin{aligned}
& \frac{C^{*}\left[\sigma, \mathrm{pk}_{1}, \mathrm{pk}_{2}, b, \mathrm{sk}\right]\left(c_{1}, c_{2}, \pi\right):}{1 . \text { Verify that } \pi \text { is a proof of }}\left(\mathrm{pk}_{1}, \mathrm{pk}_{2}, c_{1}, c_{2}\right) \in L_{E Q} \text { by running } V\left(\sigma,\left(\mathrm{pk}_{1}, \mathrm{pk}_{2}, c_{1}, c_{2}\right), \pi\right) . \\
& \quad \text { If this rejects, output } \perp . \\
& \text { 2. Compute } x=\operatorname{Decrypt}\left(\mathrm{sk}, c_{b}\right) \text {. Output } C(x) .
\end{aligned}
$$

We define the circuit garbling scheme GC $=$ (GC.circ, GC.inp, GC.eval) as follows:

- GC. $\operatorname{circ}\left(1^{\lambda}, C\right)$ : Generate $\left(\mathrm{pk}_{0}, \mathrm{sk}_{0}\right) \leftarrow \operatorname{Setup}\left(1^{\lambda}\right),\left(\mathrm{pk}_{1}, \mathrm{sk}_{1}\right) \leftarrow \operatorname{Setup}\left(1^{\lambda}\right), \sigma \leftarrow K\left(1^{\lambda}\right)$. Construct the circuit $C^{*}:=C^{*}\left[\sigma, \mathrm{pk}_{0}, \mathrm{pk}_{1}, 0, \mathrm{sk}_{0}\right]$ from $C$ as shown above. Output $\tilde{C} \leftarrow \mathcal{O}\left(1^{\lambda}, C^{*}\right)$ and $s:=\left(\sigma, \mathrm{pk}_{0}, \mathrm{pk}_{1}\right)$.

- GC.inp $(x, s)$ : Compute $c_{0} \leftarrow \operatorname{Encrypt}\left(\mathrm{pk}_{0}, x ; r_{0}\right), c_{1} \leftarrow \operatorname{Encrypt}\left(\mathrm{pk}_{1}, x ; r_{1}\right)$ and $\pi \leftarrow P\left(\sigma,\left(\mathrm{pk}_{0}, \mathrm{pk}_{1}, c_{0}, c_{1}\right),\left(r_{0}, r_{1}\right)\right)$ is a NIZK that $\left(\mathrm{pk}_{0}, \mathrm{pk}_{1}, c_{0}, c_{1}\right) \in L_{E Q}$.

Output $\tilde{x} \leftarrow\left(c_{0}, c_{1}, \pi\right)$.

- GC.eval $(\tilde{C}, \tilde{x})$ : Interpret $\tilde{C}$ as an obfuscated circuit and output $\tilde{C}(\tilde{x})$.

We note that in the above construction we need only simulation sound NIZK scheme, which does not have to provide statistical security. In particular, previously we needed statistical security to make sure that there are no inputs on which two different circuits (decrypting with $\mathbf{s k}_{0} \mathrm{vs} \mathrm{sk}_{1}$ ) differ. Now, it suffices that such inputs are hard to find.

We define a class of relevant circuit families $(\mathcal{C}$, Sam $)$ where:

- $\mathcal{C}$ consists of circuits of the form $C^{*}\left[\sigma, \mathrm{pk}_{1}, \mathrm{pk}_{2}, b, \mathrm{sk}\right]$ as described above.

- $\left(C_{0}, C_{1}, \tilde{x}_{1}, \ldots, \tilde{x}_{n}\right.$, aux $_{0}$, aux $\left._{1}\right) \leftarrow \operatorname{Sam}\left(1^{\lambda}\right)$ consists of setting $C_{0}=C^{*}\left[\sigma, \mathrm{pk}_{0}, \mathrm{pk}_{1}, b=0, \mathrm{sk}_{0}\right]$, $C_{1}=C^{*}\left[\sigma, \mathrm{pk}_{0}, \mathrm{pk}_{1}, b=1, \mathrm{sk}_{1}\right]$ where $\sigma$ is a simulated CRS chosen via $(\sigma, \tau) \leftarrow S_{1}\left(1^{\lambda}\right)$ with trapdoor $\tau$.

- The inputs $\tilde{x}_{i}=\left(c_{0}^{i}, c_{1}^{i}, \pi^{i}\right)$ consist of encryptions $c_{b}^{i} \leftarrow \operatorname{Encrypt}\left(\mathrm{pk}_{b}, x_{b}^{i}\right)$ and simulated proofs $\pi^{i} \leftarrow S_{2}\left(\sigma, \tau, c_{0}^{i}, c_{1}^{i}\right)$.

- The encrypted messages $x_{b}^{i}$ and the auxiliary information aux $x_{0}$, aux $x_{1}$ are chosen independently of $\sigma, \tau$.

Although this may seem like a complicated class of relevant circuit families, we notice that when instantiated with standard construction cryptosystem and and NIZK, the above restricted class already prevent counterexamples such as the one of [GGHW13] from going through. In particular, such counterexamples crucially rely on the auxiliary input (or the additional values $x_{i}$ ) containing and obfuscated circuit which has embedded secret information that can be used to come up with an input on which $C_{0}, C_{1}$ would distinguishably differ. In our case, the only way to come up with such an input is to know the NIZK trapdoor $\tau$ but we only give out some selected few simulated proofs $\pi^{i}$ using $\tau$ and do not provide any other information about it in any other context. It seems reasonable to postulate the existence of $\mathrm{sdiO}$ for all relevant differing-inputs families relative to some standard-construction encryption scheme $\mathcal{P K \mathcal { E }}$ and simulation sound NIZK scheme $\Pi$. 
Theorem 4.8. If $\Pi$ is simulation-sound NIZK, and $\mathcal{P K \mathcal { E }}$ is a semantically secure public-key encryption scheme and $\mathcal{O}$ is a strong differing-inputs obfuscator for relevant differing-inputs circuit families, then the above construction GC is a reusable garbled circuit with correlated distributional indistinguishability.

See Appendix F.4 for a proof of the above theorem.

\section{Analyzing Efficiency and Putting it All Together}

In the past two sections, we have shown how to construct reusable GRAM with and without persistent memory from one-time GRAM and a certain type of reusable garbled circuits. We then constructed such garbled circuits using obfuscation. So far, we have largely ignored efficiency considerations, and in this section we will analyze the efficiency of the constructions.

Overview. Recall that, on a high-level, all our constructions of reusable garbled RAM work as follows: to reusably garble a program $P$, we need to reusably garble some related circuit $C$ that computes a one-time garbled program/input $\tilde{P}_{\text {one }}, \tilde{x}_{\text {one }}$. To reusably garble $C$, we in turn need to obfuscate some related circuit $C^{*}$. On a high level, the size of $C$ and $C^{*}$ in all of the constructions scales with $\widetilde{O}(t)$, where $t$ is run-time of the program $P$. Therefore, to get the right level of efficiency for the server, we would need to rely on a reusable circuit garbling scheme where the size of a garbling of a circuit $C$ and the time it takes to evaluate it is only linear in the circuit size $O(|C|)$. This, in turn, would require us to rely on an obfuscation scheme where the size of an obfuscation of $C^{*}$ and the time it takes to evaluate it is linear is the circuit size $O\left(\left|C^{*}\right|\right)$. As we discuss in Appendix A.1, such general obfuscation schemes with linear slowdown can be conjectured to exist, but do not seem to follow directly from the basic constructions in the literature. Luckily, it turns out that we can avoid this requirement via a more careful analysis.

Bitwise Compact. We can rely on additional structure of the circuits $C, C^{*}$ which will allow us to get the right level of efficiency for the full construction without relying on general obfuscation with linear slowdown. In particular, we will rely on the fact that, although these circuits are large and have a large output size, each output bit can be computed by a much smaller circuit. We call this property bitwise compactness.

Definition 5.1 (Bitwise Compact). Let $C:\{0,1\}^{n} \rightarrow\{0,1\}^{m}$ be a function or a circuit. We say that $C$ is $s$-strongly bitwise compact if there exists a circuit $C_{b i t}:\{0,1\}^{n} \times[m] \rightarrow\{0,1\}$ of size $\left|C_{b i t}\right|=s$ such that $C_{b i t}(x, i)$ output the $i$ 'th bit of $C(x)$.

We say that $C$ is $s$-weakly bitwise compact if for each $i \in[m]$ there exists a circuit $C_{b i t, i}$ of size $\left|C_{b i t, i}\right|=s$ such that $C_{b i t, i}(x)$ outputs the $i$ 'th bit of $C(x)$.

We also require that a strongly (resp. weakly) bitwise compact circuit $C$ is given in some canonical format that makes it efficient to recover the bitwise representation $C_{b i t}$ (resp. for each $i \in[m]$, recover $\left.C_{b i t, i}\right)$ from $C$ in $\widetilde{O}(|C|)$ time..$^{8,9}$

A circuit $C$ with large output-size $m$ can be $s$-(strongly/weakly) bitwise compact where $s \ll m$ is much smaller than the output size. We will show how to use bitwise compactness to get more efficient circuit garbling and obfuscation schemes.

\footnotetext{
${ }^{8}$ For example, a canonical representation $C=\left(C_{b i t}[1], \ldots, C_{b i t}[m]\right)$ consists of $m$ parallel copies of the bitwise representation circuit with different hard-coded indices $i$.

${ }^{9}$ Note that strongly bitwise-compact implies weakly bitwise compact by defining $C_{b i t, i}(\cdot):=C_{b i t}(\cdot, i)$.
} 
Structure. We first begin by examining the efficiency of one-time GRAM from the literature in Section 5.1. Then we follow a "top down" approach, by looking at how the parameters of the reusable GRAM depend on those of the reusable circuit garbling schemes (Section 5.2), how the parameters of circuit garbling schemes depend on those of obfuscation (Section 5.3), and how we can optimize obfuscation parameter by relying on bitwise compactness (Section 5.4). Lastly, in Section 5.5, we summarize the efficiency analysis and state the parameters and assumptions needed for our constructions.

\subsection{Efficiency of One-Time GRAM}

We begin by recalling the efficiency of one-time GRAM construction of [LO13a, GHL $\left.{ }^{+} 14\right]$. We will state the parameters for the most efficient variant which uses identity-based encryption (IBE). See Appendix B for an overview of this construction and the following theorem. We mention that there is also an alternate construction which uses only one-way functions at the cost of a slight degradation in parameters.

Theorem 5.2 ([LO13a, GHL $\left.\left.{ }^{+} 14\right]\right)$. Assuming the existence of selectively-secure identity-based encryption (IBE), there exists a garbled RAM scheme GR1 = (GR1.data, GR1.prog, GR1.inp, GR1.eval) with persistent memory and one-time security. Furthermore, for a program $P$ with run-time $t$, input size $n$, and output size $m$ and memory-data of size $N$ :

- GR1.data runs in time $N \cdot \operatorname{poly}(\lambda)$

- GR1.prog can be described by a circuit of size $\widetilde{O}(t+|P|+n+m) \cdot \operatorname{poly}(\lambda, \log N)$. Furthermore, this circuit is s-strongly bitwise-compact where $s=\widetilde{O}(|P|+n+m) \cdot \operatorname{poly}(\lambda, \log N, \log t)$.

- GR1.inp can be described by a circuit of size $n \cdot \operatorname{poly}(\lambda)$.

- GR1.eval is a RAM program with run-time $\widetilde{O}(t+|P|+n+m) \cdot \operatorname{poly}(\lambda, \log N)$.

In the case without persistent memory, there is a scheme GR1 = (GR1.prog, GR1.inp, GR1.eval) that achieves the same parameters as above when we replace the memory size by $N=t$.

On a high level, the procedure GR1.prog is bitwise compact in these constructions because it essentially consists of many copies of a one-time garbled circuit (e.g., Yao) computing a single CPU step of a RAM. Each such garbled circuit can be computed very efficiently. See Appendix B for more details.

Throughout this section, it will be convenient to discuss the settings without and with persistent memory simultaneously. In this case we will follow the convention initiated in the above theorem, where any parameters involving the memory-size $N$ will apply to the setting without persistent memory by setting $N=t$.

\subsection{Efficiency of Reusable GRAM from Reusable Circuits}

Let us analyze the efficiency of the reusable garbled RAM constructions in Section 3.3 and Section 4.2 in terms of the efficiency of the underlying reusable circuit garbling scheme. Recall that these constructions show how reusably garble a RAM program $P$ by reusably garbling a related circuit $C=C[P, \ldots]$. The circuit $C$ computes a one-time garbling $\left(\tilde{P}_{\text {one }}, s_{\text {one }}\right) \leftarrow \operatorname{GR} 1 . \operatorname{prog}\left(P^{+}, \ldots\right)$, $\tilde{x}_{\text {one }} \leftarrow \mathrm{GR} 1 \cdot \operatorname{inp}(x \ldots)$. 
The Circuit $C$. Assume we start with a program $P$ with run-time $t$, input size $n$, and output size $m$ and memory-data of size $N$ (in the case without persistent memory, set $N=t$ ). To garble $P$ we construct a corresponding circuit $C$ which, in both constructions, has the following parameters:

- The size of the circuit $C$ is $q=\widetilde{O}(t+|P|+n+m) \cdot \operatorname{poly}(\lambda, \log N)$.

- The input-size of $C$ is $n^{\prime}=n+m+O(\lambda)$.

- The output-size of $C$ is $m^{\prime}=\widetilde{O}(t+|P|+n+m) \cdot \operatorname{poly}(\lambda, \log N)$.

- The circuit $C$ is $s$-strongly bitwise compact where $s=\widetilde{O}(|P|+n+m) \cdot \operatorname{poly}(\lambda, \log N, \log t)$.

The Construction. The constructions of reusable garbled RAM in Section 3.3 (without persistent memory) and Section 4.2 (with persistent memory) define the scheme GR = ([GR.data], GR.prog, GR.inp, GR.eval) in terms of the circuit $C$ and the circuit-garbling scheme GC = (GC.circ, GC.inp, GC.eval). In both cases, the efficiency parameters can be summarized as follows.

- In the setting of persistent memory, the procedure GR.data runs in time $N \cdot \operatorname{poly}(\lambda)$.

- The efficiency of GR.prog equals that of GC.circ for a circuit $C$ with the parameters above.

- The efficiency of GR.inp equals that of GC.inp for a circuit $C$ with the parameters above.

- The efficiency of GR.eval equals that of GC.eval for a circuit $C$ with the parameters above, plus an $\widetilde{O}(t+|P|+n+m) \cdot \operatorname{poly}(\lambda, \log N)$ overhead (to evaluate the one-time GRAM).

\subsection{Efficiency of Reusable Circuits from Obfuscation}

We gave three different constructions of reusable garbled circuits from obfuscation: a construction in Section 3.5 which we will call "Construction 0" and which achieves basic distributional indistinguishability, and Constructions $1 \& 2$ in Section 4.3 which achieve correlated distributional indistinguishability. All of the three constructions rely on public-key encryption, and we assume w.l.o.g. that a long message of size $\ell$ is encrypted bit-by-bit so that the complexity of encryption/decryption is $\ell$ p poly $(\lambda)$. Constructions $0 \& 2$ also rely on (SSS or SS) NIZKs for plaintext equality. Again, we assume w.l.o.g. that for a message of size $\ell$, plaintext equality is proved bit-by-bit so that the complexity of constructing and verifying such a NIZK is $\ell \cdot \operatorname{poly}(\lambda)$.

The Circuit $C^{*}$. Assume we have a circuit $C$ of size $q=|C|$, input-size $n^{\prime}$, output size $m^{\prime}$ and which is $s$-strongly bitwise compact. In all of our constructions, the problem of reusably garbling a circuit $C$ is translated into the problem of obfuscating a related circuit $C^{*}$ with the following parameters.

- In all constructions, the circuit $C^{*}$ is of size $q^{*}=q \cdot \operatorname{poly}(\lambda)$.

- In all constructions, the circuit $C^{*}$ has input size $n^{*}=n^{\prime} \cdot \operatorname{poly}(\lambda)$ and output size $m^{*}=m^{\prime}$.

- In Construction 0, the circuit $C^{*}$ is $s^{*}$-weakly bitwise-compact and in Constructions 1,2 it is $s^{*}$-strongly bitwise compact, where $s^{*}=s \cdot \operatorname{poly}(\lambda)$.

The reason that the circuit $C^{*}$ in Construction 0 is only weakly bitwise compact is that $C^{*}=$ $C^{*}\left[\sigma, \mathrm{pk}_{1}, \mathrm{pk}_{2}, b, \mathrm{sk}, u, v\right]$ has a hard-coded value $v$ which is of size $|v|=m^{\prime}$ related to the (potentially huge) output size. However, the $i$ 'th output bit of $C^{*}$ only depends on the $i$ 'th bit $v_{i}$ of $v$. In other words, there are small circuits $C_{b i t, i}^{*}=C_{b i t, i}^{*}\left[\sigma, \mathrm{pk}_{1}, \mathrm{pk}_{2}, b, \mathrm{sk}, u, v_{i}\right]$ that compute the $i$ 'th output bit of $C^{*}$. On the other hand, Constructions 1,2 do not have any hard-coded value and are strongly bitwise compact. 
The Construction(s). Let $\mathcal{O}$ be an obfuscation scheme. The parameters of $\mathrm{GC}=$ (GC.circ, GC.inp, GC.eval) in Constructions 0,1,2 can be summarized in terms of $\mathcal{O}$ as follows.

- The efficiency of GC.inp in all constructions is just $n^{\prime} \cdot \operatorname{poly}(\lambda)$.

- The efficiency of GC.circ is equal to that of obfuscating the circuit $C^{*}$ with parameters above.

- The efficiency of GC.eval is equal to that of evaluating the obfuscated circuit for $C^{*}$ with parameters above.

\subsection{Efficiency of Obfuscation}

Finally, we get to the parameters of obfuscation for a circuit $C^{*}$ of size $q^{*}$, input-size $n^{*}$, and output size $m^{*}$, which is $s^{*}$-(strongly or weakly) bitwise compact. We especially care about the setting where the circuits have huge output size, but a small compact representation: $q^{*}, m^{*} \gg s^{*}$.

Recall that we need different definitions of obfuscation for Constructions $0,1,2$, but for now we focus on efficiency consideration. Let $\mathcal{O}$ be any circuit obfuscation scheme. In general, if we only rely on the fact that $C^{*}$ is of size $q^{*}$, then the only efficiency guarantee we have on the obfuscation $\tilde{C} \leftarrow \mathcal{O}\left(1^{\lambda}, C^{*}\right)$ in terms of the run-time of $\mathcal{O}$, the size of $\tilde{C}$, and evaluation time of $\tilde{C}$, is that they are bounded by poly $\left(q^{*}, \lambda\right)$. As we discuss in Appendix A.1, we can conjecture that there are general obfuscation schemes with linear slowdown, where all of the above parameters are bounded by $q^{*} \cdot \operatorname{poly}(\lambda)$, but this does not seem to be the case for the basic constructions from the literature. In particular, it would require improved parameters for multilinear maps or stronger assumptions. Luckily, we can get improved efficiency for obfuscation when the circuit being obfuscated is bitwise compact.

Optimized Obfuscators. Let $\mathcal{O}$ be an obfuscator. We define an optimized way of obfuscating bitwise compact circuits using $\mathcal{O}$.

Definition 5.3. Let $\mathcal{O}$ be an arbitrary obfuscation scheme for circuits.

We say that an obfuscation scheme $\mathcal{O}_{w O p t}$ is weakly bitwise optimized if it works as follows. For a circuit $C^{*}$ which is $s^{*}$-weakly bitwise compact with bitwise-representation circuits $C_{\text {bit }, i}^{*}$ and output size $m^{*}$, the obfuscation $\mathcal{O}_{w O p t}\left(1^{\lambda}, C^{*}\right)$ outputs $\tilde{C}=\left[\tilde{C}_{1}, \ldots, \tilde{C}_{m^{*}}\right]$ where $\tilde{C}_{i} \leftarrow \mathcal{O}\left(1^{\lambda}, C_{\text {bit }, i}^{*}\right)$.

We say that an obfuscation scheme $\mathcal{O}_{\text {sopt }}$ is strongly bitwise optimized if it works as follows. For any circuit $C^{*}$ which is $s^{*}$-strongly bitwise compact and has bitwise-representation $C_{\text {bit }}^{*}$ and output size $m^{*}$, the obfuscation $\mathcal{O}_{\text {sOpt }}\left(1^{\lambda}, C^{*}\right)$ outputs $\tilde{C} \leftarrow \mathcal{O}\left(1^{\lambda}, C_{\text {bit }}^{*}\right)$. To evaluate $\tilde{C}$ on input $x$, evaluate $\left[\tilde{C}(x, 1), \ldots, \tilde{C}\left(x, m^{*}\right)\right]$.

Note that for a weakly bitwise optimized scheme, the time it takes to create $\tilde{C}$ and its size are bounded by $m^{*} \cdot \operatorname{poly}\left(s^{*}, \lambda\right)$. In a strongly optimized scheme this is reduced to poly $\left(s^{*}, \lambda\right)$ and independent of $m^{*}$. In particular, notice that the obfuscated representation $\tilde{C}$ can be smaller than the original circuit $C^{*}{ }^{10}$ In both cases, the time it takes to evaluate the obfuscated circuit $\tilde{C}$ on an input $x$ is $m^{*} \cdot \operatorname{poly}\left(s^{*}, \lambda\right)$. These parameters hold if we assume arbitrary polynomial efficiency of the underlying obfuscator $\mathcal{O}$. If we further assume that the underlying obfuscator $\mathcal{O}$ has linear slowdown, all of the above factors of poly $\left(s^{*}, \lambda\right)$ become $s^{*} \cdot \operatorname{poly}(\lambda)$.

\footnotetext{
${ }^{10}$ Technically speaking, $\tilde{C}$ is not in itself a circuit that evaluates $C^{*}$. However, it provides all of the information needed to evaluate $C^{*}$ at any input.
} 
Can we Assume Obfuscator is Optimized? In the case of indistinguishability obfuscation (iO) the obfuscation schemes $\mathcal{O}_{w O p t}$ and $\mathcal{O}_{s O p t}$ satisfy the definition of iO whenever $\mathcal{O}$ does, with the following caveat: in the security definition, instead of just requiring that the circuits $C_{0}^{*}, C_{1}^{*}$ are of the same size $\left|C_{0}^{*}\right|=\left|C_{1}^{*}\right|$, we now also require that their strongly/weakly bitwise representations are also of the same size. ${ }^{11}$ In particular, we rely on the fact that if the two circuits $C_{0}^{*}, C_{1}^{*}$ compute the same function than so do their bitwise representations.

In the case of strong differing inputs obfuscation (sdiO) the strongly bitwise optimized scheme $\mathcal{O}_{s O p t}$ satisfies sdiO security for a differing inputs family $\left(C^{0}, C^{1}, x_{1}, \ldots, x_{n}\right.$, aux $x_{0}$, aux 1$) \leftarrow \operatorname{Sam}\left(1^{\lambda}\right)$ as long as $\mathcal{O}$ satisfies sdiO security for a closely related differing inputs family $\left(C_{b i t}^{0}, C_{b i t}^{1},\left\{\left(x_{i}, j\right): i \in\right.\right.$ $\left.[n], j \in[m]\}, \operatorname{aux}_{0}, \operatorname{aux}_{1}\right) \leftarrow \operatorname{Sam}^{\prime}\left(1^{\lambda}\right)$ where $C_{b i t}^{b}$ denotes the bitwise representation of $C^{b}$. Therefore, assuming sdiO security of the strongly optimized scheme $\mathcal{O}_{s O p t}$ is conceptually not a very different assumption. ${ }^{12}$

In the case of the obfuscation conjecture (Conjecture 4.5) used in Construction 1, we can simply augment the conjecture to hold for a strongly or weakly optimized obfuscation scheme $\mathcal{O}_{s O p t}$ or $\mathcal{O}_{w O p t}$, but there is no simple reduction to show that this is implies by the security of $\mathcal{O}$. Still, such an augmented conjecture does not seem conceptually very different from the original form.

\subsection{Summary}

We summarize the discussion from the above sections and state the final parameters and assumptions of our schemes.

Without Persistent Memory. Let us start with the case without persistent memory, relying on indistinguishability obfuscation. Following the above discussion, the problem of garbling a program $P$ translates to reusably garbling some related strongly bitwise compact circuit $C$ with (basic) distributional indistinguishability, which translates to $\mathrm{iO}$ for some weakly bitwise compact circuit $C^{*}$ via Construction 0 in Section 3.5. We assume w.l.o.g. that the iO scheme is weakly bitwise optimized.

Combining all of the above and the facts that indistinguishability obfuscation + one-way function implies selectively secure functional encryption which implies selectively secure IBE [GGH $\left.{ }^{+} 13 \mathrm{~b}\right]$, and that statistically simulation sound NIZK can be constructed from statistically sounds NIZK $\left[\mathrm{GGH}^{+} 13 \mathrm{~b}\right]$, we get the following theorem.

Theorem 5.4. If indistinguishability obfuscation (iO), one-way functions, and statistically sounds NIZKs exist, then there exists a reusable garbled-RAM scheme without persistent memory satisfying Definition 3.2. Furthermore, it supports public input garbling. For a program $P$ with run-time bound $t$, input size $n$, and output size $m$ it achieves the following efficiency:

- GR.inp runs in time $(m+n) \cdot \operatorname{poly}(\lambda)$.

- GR.prog and GR.eval run in time $\widetilde{O}(t) \cdot \operatorname{poly}(\lambda+n+m+|P|)$. If we further assume $i O$ with linear slowdown, then GR.prog and GR.eval run in time $\widetilde{O}(t+|P|+n+m) \cdot \operatorname{poly}(\lambda)$.

An alternate construction of reusable garbled RAM without persistent memory from functional encryption appears in Section D.

\footnotetext{
${ }^{11}$ In our case, the circuits are the same up to the setting of some hard-coded flag, and therefore this requirement is satisfied.

${ }^{12}$ Interestingly, it is not clear if the same holds for a weakly optimized scheme.
} 
With Persistent Memory, No Preprocessing. In the case of persistent memory, the problem of garbling a program $P$ translates to reusably garbling some related strongly bitwise-compact circuit $C$. The garbling now needs to satisfy the stronger notion of correlated distributional indistinguishability. This translates to obfuscating a strongly bitwise compact circuit $C^{*}$ via Constructions 1 or 2 in Section 4.3. Note that we can also use Constructions 1,2 in the case without persistent memory to get better efficiency, since correlated distributional indistinguishability clearly implies basic distributional indistinguishability. In particular, the advantage of Constructions 1 or 2 is that the resulting circuit $C^{*}$ is strongly bitwise compact and therefore can be obfuscated very efficiently. This allows us to get rid of pre-processing - the time it takes to garble a program $P$ is small and essentially independent in the program's run-time $t$. Summarizing the discussion, we get the following theorem.

Theorem 5.5. Assume the existence of selectively secure IBE schemes, statistically sound NIZKs and a strongly bitwise optimized obfuscator $\mathcal{O}_{\text {sOpt }}$ which either (1) satisfies Conjecture 4.5, or (2) satisfies sdiO security for relevant circuit families (Definition 4.7). ${ }^{13}$ Then there exist reusable garbled-RAM schemes both with and without persistent memory. For a program $P$ with run-time bound $t$, input size $n$, output size $m$, and memory-data size $N$ (in the case without persistent data set $N=t$ ) it achieves the following efficiency:

- In the case of persistent data, the procedure GR.data runs in time $N \cdot \operatorname{poly}(\lambda)$.

- GR.inp runs in time $(m+n) \cdot \operatorname{poly}(\lambda)$.

- GR.prog runs in time poly $(\lambda, \log t, \log N,|P|, n, m)$ and, in particular, is sub-linear in $t$. If we further assume that the underlying obfuscator has linear slowdown then GR.prog runs in time $\widetilde{O}(|P|+n+m)$ poly $(\lambda, \log t, \log N)$.

- GR.eval run in time $\widetilde{O}(t) \operatorname{poly}(\lambda, \log N,|P|, n, m)$. If we further assume that the underlying obfuscator has linear slowdown then GR.eval run in time $\widetilde{O}(t+|P|+n+m) \operatorname{poly}(\lambda, \log N)$.

\section{$6 \quad$ Extensions and Applications}

Program Privacy. Our default definitions of garbled RAM do not include program privacy, and the garbled program $\tilde{P}$ may reveal information about the code of the actual program $P$ to the server. There are several simple standard techniques that can be employed to add program privacy to our constructions. Firstly, we can garble a program $P_{u n i}$ which is the universal RAM that runs for some fixed number of steps $t$. The code of the actual program $P$ that we want to execute can then be provided as part of the input to $P_{u n i}$ or, in the case of persistent memory, it can be included as part of the initial memory data $D$. Alternatively, to avoid sending the code of the program $P$ with each input in the case without persistent memory, we can use the following approach of $\left[\mathrm{GKP}^{+} 13 \mathrm{~b}\right]$ : Instead of garbling the program $P$, we encrypt it to get a cipehrtext $c_{P}=\operatorname{Enc}_{k}(P)$. We then garble a program $Q_{e n c}\left[c_{P}\right]$ that has $c_{P}$ hard wired in it, and on input $(k, x)$ it decrypts $c_{P}$ with key $k$, interpret the result as a program, and run that program on input $x$. Notice that the input size for $Q_{e n c}\left[c_{P}\right]$ is independent of the size of $P$ itself, and that the description of $Q_{e n c}\left[c_{P}\right]$ does not reveal anything about the actual program $P$.

Output Privacy. Our default definition of garbled RAM assumes that the server who evaluates the garbled program on the garbled input learns the output $y$ of the program. This might be useful in some scenarios, but in other cases the output $y$ is intended for the client and the server simply

\footnotetext{
${ }^{13}$ The latter is implied by the existence of any sdiO obfuscator $\mathcal{O}$ for a related class of circuit families, as discussed.
} 
sends it back to the client. In such cases, we may also want the output $y$ of the program to remain secret from the server and only be revealed to the client. We can achieve output privacy by simply garbling an augmented program $P_{\text {outEnc }}$ which gets as input $(k, x)$, evaluates $y=P(x)$ and outputs an encryption $c=\operatorname{Enc}_{k}(y)$, where (Enc, Dec) is some (one-time) encryption scheme (e.g., one-time pad). The client garbles inputs $\left(x_{i}, k_{i}\right)$ where $k_{i}$ is a fresh key for encryption, the server evaluates the garbled program on the garbled input to get back $c_{i}=\operatorname{Enc}_{k_{i}}\left(y_{i}\right)$ where $y_{i}=P\left(x_{i}\right)$, sends $c_{i}$ to the client, and the client decrypts $y_{i}=\operatorname{Dec}_{k_{i}}\left(c_{i}\right)$. Notice that the entire view of the server can be simulated given the values $c_{i}$ and therefore the server learns nothing about the inputs $x_{i}$ or the outputs $y_{i}$ of the program executions.

Verifiable Computation. In the above scenario, where the program outputs $y_{i}$ are intended for the client, we may also want to add verifiability, where the client is sure that the received outputs $y_{i}$ are indeed the correct outputs of the computation. To do so, we can garble an augmented program $P_{\text {outAuth }}$ which gets as input $(k, x)$, evaluates $y=P(x)$ and outputs $(y, \sigma)$ where $\sigma$ is an authentication-tag $\sigma=\operatorname{MAC}_{k}(y)$ for some (one-time) message-authentication code MAC. The client garbles inputs $\left(x_{i}, k_{i}\right)$ where $k_{i}$ is a fresh key for the MAC, the server evaluates the garbled program on the garbled input to get back $\left(y_{i}, \sigma_{i}\right)$ which it sends to the client, and the client verifies $\sigma_{i}=\operatorname{MAC}_{k_{i}}\left(y_{i}\right)$. Notice that the entire view of the server can be simulated given the values $\left(y_{i}, \sigma_{i}\right)$ and therefore the server cannot come up with a valid tag $\sigma_{i}^{\prime}$ for any $y_{i}^{\prime} \neq y_{i}$. This gives us verifiable computation. Furthermore, we can always combine privacy and authentication by garbling an augmented program $P_{\text {outEncAuth }}$ which encrypts the output and then authenticates the ciphertext.

Input-Specific Run-Time. Our default notion of garbled RAM assumes that the program $P$ has some fixed worst-case run-time $t=t(n)$ for all inputs $x$ of size $n$, and and the running time of the server during each evaluation is proportional to $t$. However, a program might have vastly different run-times for different inputs, and we would like the server's work when evaluating the garbled program $\tilde{P}$ on garbled inputs $\tilde{x}$ to only depend on the input-specific run-time of $P(x)$ rather than the worst-case run-time $t$. Of course, this means that we have to leak the input-specific run-time of $P(x)$, but the goal is to only leak this information and nothing else. The works of $\left[\mathrm{GKP}^{+} 13 \mathrm{~b}, \mathrm{GKP}^{+} 13 \mathrm{~b}\right]$ show how to do this in the case of Turing Machine computation, where the server's run-time is proportional to the input-specific Turing-Machine run-time, and we can use some of the same techniques to get analogous results for RAM computation.

In the setting without persistent memory, the high-level idea goes as follows. To garble a program $P$, we separately garble $\ell=\log (t)$ programs $P_{1}, P_{1}, \ldots, P_{\ell}$ where each program $P_{j}(x)$ runs $P(x)$ for $2^{j}$ steps, and if the computation completes returns the output else indicates that the computation is incomplete. The server gets these independently garbled programs $\tilde{P}_{1}, \ldots, \tilde{P}_{\ell}$. To garble an input $x$, the client just creates $\ell$ independently garbled inputs $\tilde{x}_{j}$ for each of the reusable garbled RAM programs $\tilde{P}_{j}$ and the server evaluates them one by one (starting with $j=1$ ) until the first one terminates. If the input-specific run-time is $t_{x}$ then the time to evaluate all of the additional programs only introduces a constant amount of overhead and therefore the server's run-time is $\widetilde{O}\left(t_{x}\right)$. Notice that if we use a garbled RAM scheme with parameters as in Theorem 5.5, then the client never does work proportional to $t$ but only proportional to $\log (t)$ where $t$ is the worst-case run-time

In a setting with persistent memory, the solution becomes more complex for several reasons:

- The most serious issue is that our solution of reusable GRAM with persistent memory only allows us to garble a single program per database, and moreover we assume that this program always takes the same number of steps to run (since we are garbling a circuit whose output length depends on the run-time of the program). This issue can be addressed by using the 
"strongly bitwise compact" representation (see Section 5). In particular, we can reusably garble a single circuit $C[P, \ldots]$ with a similar description as before but taking an additional input $t_{\text {cur }} \in\{1, \ldots, t\}$ and outputting a variable-length output consisting of $(\tilde{P}, \tilde{x})$ where $\tilde{P}$ runs for $t_{c u r}$ steps. In particular, this circuit is "strongly bitwise-compact" even if the total number of output bits is not fixed. The client can sequentially garble her input with the values $t_{c u r}=2^{j}$ for $j=1, \ldots, \log t$ until she hits a value $t_{c u r}$ for which the computation completes. Several minor issues remain.

- One issue is that each program execution which does not complete must "clean up after itself." Namely if execution does not halt within $t_{c u r}$ steps then it needs to restore the memory to its original state. This can be done without increasing the running time by too much.

- Yet another problem is that when garbling the $i$ 'th input, the client must know the total number of CPU steps executed so far (see generalized definition of one-time garbled RAM in $\left.\left[\mathrm{GHL}^{+} 14\right]\right)$. Here we must revert to an interactive solution, where the client garbles these inputs one at a time and waits for the server to tell it whether the computation completed before it garbles the same input again (or a different input if the computation was completed).

The above outlines the high-level ideas and we defer a formal treatment of this to future work.

Applications to MPC. We have discussed garbled RAM in the context of outsourcing computation where the program specification, persistent memory/databse, and inputs are all chosen by a single client. However, we can also employ garbled RAM in the setting of two-party or multiparty computation (MPC) where these values belong to multiple parties. In particular, we can run standard MPC protocols to garble memory data $D$ and program $P$ where the underlying data belongs to several mutually distrustful parties. Later, the parties can run MPC protocol(s) to create garbled inputs $x_{i}$, which may also depend on the inputs of several parties. The main advantage is that there can now be one designated (and untrusted) party that does the work of evaluating the garbled program on the garbled input in time proportional to the RAM computation, and all other parties only need to work in time proportional to the input size. This is in contrast to standard MPC approaches where all parties work as hard as the circuit size of the program evaluation, or the work of $\left[\mathrm{GKK}^{+} 12\right]$ where all parties work as hard as the RAM complexity of the computation.

Functional Encryption for RAMs. In Appendix D, we also show how to extend our techniques to get functional encryption for RAMs (without persistent memory). In such a scheme, secret keys are associated with functions $f$. If a user gets a secret keys $\mathrm{SK}_{f_{1}}, \ldots, \mathrm{SK}_{f_{q}}$ for functions $f_{1}, \ldots, f_{q}$ and a ciphertext $c$ encrypting some message $x$, she should only learn $f_{1}(x), \ldots, f_{q}(x)$ and nothing else. Unfortunately, it is known that simulation based security cannot be achieved in this setting if an attacker can get secret keys for arbitrarily many different functions (see [AGVW13]), and therefore we must settle for indistinguishability based security. Prior work of [GGH $\left.{ }^{+} 13 \mathrm{~b}\right]$ showed how to construct such functional encryption for circuits, where the size of the secret key and the decryption time were proportional to the circuit size of $f$. We essentially show how to convert any such functional encryption scheme for circuits into a functional encryption for RAMs where the size of the secret keys and the decryption time are proportional to the RAM run-time of $f$. In particular, we get such constructions assuming indistinguishability obfuscation. We also show how to convert any such FE for RAMs (with indistinguishability security) into a reusable garbled RAM (with simulation security) and therefore this gives us an alternate construction of reusable garbled RAM from FE for circuits. 
Obfuscation for RAM programs. In Appendix E, we propose a candidate scheme that converts an obfuscator for circuits into an obfuscator for RAM programs. In particular, the size of the obfuscated program is only proportional to the size of the original program and the running time of the obfuscated program is only proportional to the (worst-case) running time of the original RAM program. We merely conjecture the security of the construction but do not have a reduction from any simple-to-state assumption.

\section{Conclusions}

We have shown how to privately outsource RAM computation from a weak client to a more powerful server via reusable garbled RAM schemes. Our main contribution was to reduce the problem of reusable garbled RAM into seemingly simpler problems dealing with reusable garbled circuits. In doing so, we introduced new notions of security for such garbled circuit that we call "distributional indistinguishability" and "correlated distributional indistinguishability" which may be of independent interest and seem to allow for greater (output-size independent) efficiency than the stronger simulation-based security. Lastly, we showed how to construct such schemes under obfuscationbased assumptions. The main open problem is to provide constructions of such reusable garbled circuits under weaker assumptions. Ideally, such constructions would avoid obfuscation altogether, but a more limited goal would be to get "correlated distributional indistinguishability" from indistinguishability obfuscation.

\section{Acknowledgments}

The authors would like to thank Yael Tauman Kalai, Nir Bitansky and Omer Paneth for enlightening initial discussions on the topics of this work.

\section{References}

$\left[\mathrm{ABG}^{+} 13\right]$ Prabhanjan Ananth, Dan Boneh, Sanjam Garg, Amit Sahai, and Mark Zhandry. Differing-inputs obfuscation and applications. Cryptology ePrint Archive, Report 2013/689, 2013. http://eprint.iacr.org/.

$\left[\mathrm{AFK}^{+} 14\right]$ Daniel Apon, Xiong Fan, Jonathan Katz, Feng-Hao Liu, Elaine Shi, and Hong-Sheng Zhou. Non-interactive cryptography in the ram model of computation. Cryptology ePrint Archive, Report 2014/154, 2014. http://eprint.iacr.org/.

[AGVW13] Shweta Agrawal, Sergey Gorbunov, Vinod Vaikuntanathan, and Hoeteck Wee. Functional encryption: New perspectives and lower bounds. In Canetti and Garay [CG13], pages $500-518$.

[Bar86] D A Barrington. Bounded-width polynomial-size branching programs recognize exactly those languages in $n c_{1}$. In $S T O C, 1986$.

[BCCT13] Nir Bitansky, Ran Canetti, Alessandro Chiesa, and Eran Tromer. Recursive composition and bootstrapping for snarks and proof-carrying data. In Boneh et al. [BRF13], pages 111-120.

[BCP13] Elette Boyle, Kai-Min Chung, and Rafael Pass. On extractability obfuscation. Cryptology ePrint Archive, Report 2013/650, 2013. http://eprint.iacr.org/. 
[BFR $\left.{ }^{+} 13\right]$ Benjamin Braun, Ariel J. Feldman, Zuocheng Ren, Srinath T. V. Setty, Andrew J. Blumberg, and Michael Walfish. Verifying computations with state. IACR Cryptology ePrint Archive, 2013:356, 2013.

[BGI $\left.{ }^{+} 12\right]$ Boaz Barak, Oded Goldreich, Russell Impagliazzo, Steven Rudich, Amit Sahai, Salil P. Vadhan, and Ke Yang. On the (im)possibility of obfuscating programs. J. ACM, $59(2): 6,2012$.

[BGK $\left.{ }^{+} 13\right]$ Boaz Barak, Sanjam Garg, Yael Tauman Kalai, Omer Paneth, and Amit Sahai. Protecting obfuscation against algebraic attacks. IACR Cryptology ePrint Archive, 2013:631, 2013.

[BR13] Zvika Brakerski and Guy N. Rothblum. Virtual black-box obfuscation for all circuits via generic graded encoding. IACR Cryptology ePrint Archive, 2013:563, 2013.

[BRF13] Dan Boneh, Tim Roughgarden, and Joan Feigenbaum, editors. Symposium on Theory of Computing Conference, STOC'13, Palo Alto, CA, USA, June 1-4, 2013. ACM, 2013.

[BSCG $\left.^{+} 13\right]$ Eli Ben-Sasson, Alessandro Chiesa, Daniel Genkin, Eran Tromer, and Madars Virza. Snarks for c: Verifying program executions succinctly and in zero knowledge. In Canetti and Garay [CG13], pages 90-108.

[BSCGT13] Eli Ben-Sasson, Alessandro Chiesa, Daniel Genkin, and Eran Tromer. Fast reductions from rams to delegatable succinct constraint satisfaction problems: extended abstract. In Robert D. Kleinberg, editor, ITCS, pages 401-414. ACM, 2013.

[CG13] Ran Canetti and Juan A. Garay, editors. Advances in Cryptology - CRYPTO 2013 33rd Annual Cryptology Conference, Santa Barbara, CA, USA, August 18-22, 2013. Proceedings, Part II, volume 8043 of Lecture Notes in Computer Science. Springer, 2013.

[CIJ ${ }^{+}$13] Angelo De Caro, Vincenzo Iovino, Abhishek Jain, Adam O’Neill, Omer Paneth, and Giuseppe Persiano. On the achievability of simulation-based security for functional encryption. In CRYPTO, 2013.

[CKGS98] Benny Chor, Eyal Kushilevitz, Oded Goldreich, and Madhu Sudan. Private information retrieval. J. ACM, 45(6):965-981, 1998.

[CR73] Stephen A. Cook and Robert A. Reckhow. Time bounded random access machines. J. Comput. Syst. Sci., 7(4):354-375, 1973.

[CS98] Ronald Cramer and Victor Shoup. A practical public key cryptosystem provably secure against adaptive chosen ciphertext attack. In Hugo Krawczyk, editor, CRYPTO, volume 1462 of Lecture Notes in Computer Science, pages 13-25. Springer, 1998.

[FLS99] Uriel Feige, Dror Lapidot, and Adi Shamir. Multiple non-interactive zero knowledge proofs under general assumptions. SIAM Journal of Computing, 29(1):1-28, 1999.

[Gen09] Craig Gentry. Fully homomorphic encryption using ideal lattices. In STOC, pages 169-178, 2009.

[GGH13a] Sanjam Garg, Craig Gentry, and Shai Halevi. Candidate multilinear maps from ideal lattices. In EUROCRYPT, 2013. 
[GGH+13b] Sanjam Garg, Craig Gentry, Shai Halevi, Mariana Raykova, Amit Sahai, and Brent Waters. Candidate indistinguishability obfuscation and functional encryption for all circuits. volume 2013, page 451, 2013. To appear in FOCS 2013.

[GGHW13] Sanjam Garg, Craig Gentry, Shai Halevi, and Daniel Wichs. On the implausibility of differing-inputs obfuscation and extractable witness encryption with auxiliary input. Cryptology ePrint Archive, Report 2013/860, 2013. http://eprint.iacr.org/.

$\left[\mathrm{GHL}^{+} 14\right]$ Craig Gentry, Shai Halevi, Steve Lu, Rafail Ostrovsky, Mariana Raykova, and Daniel Wichs. Garbled ram revisited. EUROCRYPT, 2014.

[GHRW14] Craig Gentry, Shai Halevi, Mariana Raykova, and Daniel Wichs. Garbled ram revisited, part i. Cryptology ePrint Archive, Report 2014/082, 2014. http://eprint. iacr.org/.

$\left[\mathrm{GKK}^{+} 12\right]$ S. Dov Gordon, Jonathan Katz, Vladimir Kolesnikov, Fernando Krell, Tal Malkin, Mariana Raykova, and Yevgeniy Vahlis. Secure two-party computation in sublinear (amortized) time. In CCS, 2012.

$\left[\mathrm{GKP}^{+} 13 \mathrm{a}\right]$ Shafi Goldwasser, Yael Tauman Kalai, Raluca A. Popa, Vinod Vaikuntanathan, and Nickolai Zeldovich. How to run turing machines on encrypted data. In Canetti and Garay [CG13], pages 536-553.

$\left[\mathrm{GKP}^{+} 13 \mathrm{~b}\right]$ Shafi Goldwasser, Yael Tauman Kalai, Raluca A. Popa, Vinod Vaikuntanathan, and Nickolai Zeldovich. Reusable garbled circuits and succinct functional encryption. In Boneh et al. [BRF13], pages 555-564.

[GO96] Oded Goldreich and Rafail Ostrovsky. Software protection and simulation on oblivious rams. J. ACM, 43(3):431-473, 1996.

[LO13a] Steve Lu and Rafail Ostrovsky. How to garble ram programs. In Thomas Johansson and Phong Q. Nguyen, editors, EUROCRYPT, volume 7881 of Lecture Notes in Computer Science, pages 719-734. Springer, 2013.

[LO13b] Steve Lu and Rafail Ostrovsky. How to garble RAM programs. Presentation in TCC 2013 rump session, 2013.

[LO14] Steve Lu and Rafail Ostrovsky. Garbled ram revisited, part ii. Cryptology ePrint Archive, Report 2014/083, 2014. http://eprint.iacr.org/.

[OS97] Rafail Ostrovsky and Victor Shoup. Private information storage. In STOC, 1997.

[PF79] Nicholas Pippenger and Michael J. Fischer. Relations among complexity measures. $J$. ACM, 26(2):361-381, 1979.

[PTS13] Rafael Pass, Sidharth Telang, and Karn Seth. Obfuscation from semantically-secure multi-linear encodings. IACR Cryptology ePrint Archive, 2013:781, 2013.

[RAD78] Ron Rivest, Leonard Adleman, and Michael L. Dertouzos. On data banks and privacy homomorphisms. In Foundations of Secure Computation, pages 169-180, 1978.

[Val08] Paul Valiant. Incrementally verifiable computation or proofs of knowledge imply time/space efficiency. In Ran Canetti, editor, TCC, volume 4948 of Lecture Notes in Computer Science, pages 1-18. Springer, 2008. 
[Yao82] Andrew Chi-Chih Yao. Protocols for secure computations (extended abstract). In FOCS, pages 160-164, 1982.

\section{A Additional Preliminary Definitions}

\section{A.1 Obfuscation}

Definition A.1 (Indistinguishability Obfuscator $(i \mathcal{O})$ ). A uniform PPT machine $i \mathcal{O}$ is called an indistinguishability obfuscator if the following conditions are satisfied:

- For all security parameters $\lambda \in \mathbb{N}$, for all $C \in \mathcal{C}_{\lambda}$, for all inputs $x$, we have that

$$
\operatorname{Pr}\left[C^{\prime}(x)=C(x): C^{\prime} \leftarrow i \mathcal{O}\left(1^{\lambda}, C\right)\right]=1
$$

- For any (not necessarily uniform) PPT distinguisher $D$, there exists a negligible function $\alpha$ such that the following holds: For all security parameters $\lambda \in \mathbb{N}$, for all circuit families $C_{0}=\left\{C_{\lambda}^{0}\right\}_{\lambda \in \mathbb{N}}, C_{1}=\left\{C_{\lambda}^{1}\right\}_{\lambda \in \mathbb{N}}$ of size $\left|C_{\lambda}^{0}\right|=\left|C_{\lambda}^{1}\right|$, we have that if $C_{\lambda}^{0}(x)=C_{\lambda}^{1}(x)$ for all inputs $x$, then

$$
\left|\operatorname{Pr}\left[D\left(i \mathcal{O}\left(1^{\lambda}, C_{\lambda}^{0}\right)\right)=1\right]-\operatorname{Pr}\left[D\left(i \mathcal{O}\left(1^{\lambda}, C_{\lambda}^{1}\right)\right)=1\right]\right| \leq \alpha(\lambda)
$$

The work of Garg et al. [GGH $\left.{ }^{+} 13 \mathrm{~b}\right]$ presents a candidate construction for indistinguishability obfuscation based on a new assumption regarding multilinear maps. Followup works of $\left[\mathrm{BR} 13, \mathrm{BGK}^{+} 13, \mathrm{PTS} 13\right]$ give variants of this construction that can be proven secure in the generic multilinear group model and/or under simpler-to-state assumptions.

Linear Slowdown. Our efficiency analysis in Section 5 would be simplified and the parameters would be slightly improved if we assumed that an $\mathrm{iO}$ scheme has linear slowdown. This means that the run-time of $C^{\prime} \leftarrow i \mathcal{O}\left(1^{\lambda}, C\right)$ is bounded by $|C| \cdot \operatorname{poly}(\lambda)$ and this also serves as a bound on the size of the obfuscated circuit $\left|C^{\prime}\right|$. We now explore this assumption and how it fits in with known constructions.

The main approach to constructing iO in all prior work $\left[\mathrm{GGH}^{+} 13 \mathrm{~b}, \mathrm{BR} 13, \mathrm{BGK}^{+} 13\right.$, PTS13] consists of two steps:

Circuit to Branching-Program Conversion: The first step reduces the problem of obfuscating an arbitrary polynomial-time circuit $C$ to the problem of obfuscating a constant-width polynomial-size branching program (BP). One can decompose this into two further sub-steps.

The first sub-step reduces the problem of obfuscating $C$ to that of obfuscating some "shallow" circuit $C^{\prime}$. The circuit $C^{\prime}$ gets as input $O(|C|)$ ciphertexts under a fully homomorphic encryption. It first performs $O(|C|)$ "consistency checks" on various triples of ciphertexts, where it checks that the third ciphertext in the tripple is a result of a homomorphic operation on the first two, and if all of these checks pass, it then decrypts one of the ciphertext. The "consistency checks" and the "decryption" procedures can be expressed as sub-circuits with fan-in-2 and depth $O(\log \lambda)$. The circuit $C^{\prime}$ can be expressed as an AND of these $O(|C|)$ sub-circuits (if all the checks pass, then the output is the decrypted bit, else the output is 0 ).

The second sub-step relies on Barrington's theorem [Bar86] to transform $C^{\prime}$ into a constantwidth, polynomial-size BP. Unfortunately, since $C^{\prime}$ has depth $O(\log \lambda+\log |C|)$ using fan-in-2 gates, a naive application of Barrington's theorem to $C^{\prime}$ directly would result in a $\mathrm{BP}$ of size 
$O\left(|C|^{2}\right)$ poly $(\lambda)$. However, there is a better approach to transforming $C^{\prime}$ into a BP, relying on the fact that it consists of an AND of $O(|C|)$ shallow sub-circuits. We only apply Barrington's theorem on each of the sub-circuits separately to convert each of them into a width-5 BP of size poly $(\lambda)$. We can then compute the AND of $O(|C|)$ different width-5, poly $(\lambda)$-size BPs using a width- $6 \mathrm{BP}$ of size $O(|C|) \cdot$ poly $(\lambda)$. In particular, the new BP runs the underlying component BPs sequentially but has an extra state to indicate that the current AND of the BPs executed so far is 0 . If any of the component BPs evaluates to 0, the combined BP just goes to the 0 state and stays there. To summarize, with the above modification, we reduce the problem of obfuscating an arbitrary polynomial-time circuit $C$ to the problem of obfuscating a width-6 BP of size $O(|C|)$ poly $(\lambda)$.

Obfuscating Branching Programs: The second step provides a way to obfuscate BPs using multilinear maps. The high-level idea is to encode the matrices that define the BP in the exponent. There are several variants of this approach. The initial work of Garg et al. $\left[\mathrm{GGH}^{+} 13 \mathrm{~b}\right]$ used a somewhat more complex approach that was simplified by subsequent works [BR13, $\mathrm{BGK}^{+} 13$, PTS13]. In particular, these latter results take a BP of size $n$ and create an obfuscate circuit consisting of $O(n)$ group elements. Unfortunately, for the known constructions of multilinear maps in [GGH13a], the size of the group elements themselves depends on the degree of multilinearity which depends on $n$.

Summarizing the above, we would get obfuscation with linear slowdown if we assumed the existence of multilinear maps where the size of the group elements would be some fixed polynomial in the security parameter and unrelated to the amount of multilinearity. Unfortunately, we do not have such candidates.

An Alternate Approach to Linear Slowdown. We also note that we can use ideas similar to those of $\left[\mathrm{ABG}^{+} 13, \mathrm{BCP} 13\right]$ to construct candidate obfuscation schemes with linear slowdown. Instead of obfuscating a circuit $C$ directly, we can do the following:

- Provide two encryptions of $C$ under different FHE schemes.

- Obfuscate a circuit $C^{\prime}$ which contains a hash $\sigma$ of the encryption of $C$ and the secret key for one of the FHE schemes. It gets as input two ciphertexts and a succinct proof (succinct-noninteractive argument of knowledge, SNARK) that they were both computed by homomorphically by evaluating an encrypted circuit $C$ where the hash of the encryptions is $\sigma$ on some input $x$. If the proof verifies then $C^{\prime}$ decrypts one of the ciphertexts.

The size of the circuit $C^{\prime}$ is only poly $(\lambda)$ and independent of that of $C$. Moreover the resulting scheme can be shown to satisfy iO is the obfuscation of $C^{\prime}$ satisfies differing input obfuscation (diO). Therefore, this requires stronger assumptions.

\section{A.2 Statistically Simulation-Sound Non-Interactive Zero Knowledge (NIZK)}

Another primitive introduced by $\left[\mathrm{GGH}^{+} 13 \mathrm{~b}\right]$, which we will use, is statistically simulation sound NIZK. It was shown that such NIZKs can be constructed from standard NIZKs and a commitment scheme.

Definition A.2. A statistically simulation-sound non-interactive zero-knowledge proof $(K, P, V)$ for a relation $R$ has the following properties:

Perfect COmpleteness. A proof system is complete if an honest prover with a valid witness can convince an honest verifier. Formally we have

$$
\operatorname{Pr}\left[\sigma \leftarrow K\left(1^{\lambda}\right): \exists(x, \pi): x \notin L: V(\sigma, x, \pi)=1\right]=1 .
$$


Statistical soundness. A proof system is sound if it is infeasible to convince an honest verifier when the statement is false. For all (even unbounded) adversaries $\mathcal{A}$ we have

$$
\operatorname{Pr}\left[\sigma \leftarrow K\left(1^{\lambda}\right) ;(x, \pi) \leftarrow \mathcal{A}(\sigma): V(\sigma, x, \pi)=1: x \notin L\right]=\operatorname{neg}(\lambda) .
$$

Computational ZERO-KNOWLEdGe [FLS99]. A proof system is computational zero-knowledge if the proofs do not reveal any information about the witnesses to a bounded adversary. We say a non-interactive proof $(K, P, V)$ is computational zero-knowledge if there exists a polynomial time simulator $S=\left(S_{1}, S_{2}\right)$, where $S_{1}$ returns a simulated common reference string $\sigma$ together with a simulation trapdoor $\tau$ that enables $S_{2}$ to simulate proofs without access to the witness. For all non-uniform polynomial time adversaries $\mathcal{A}$ we have for all $x \in L$

$$
\operatorname{Pr}\left[\mathcal{A}(x, \sigma, \pi)=1: \begin{array}{c}
\sigma \leftarrow K\left(1^{\lambda}\right) \\
\pi \leftarrow P(\sigma, x, w)
\end{array}\right] \approx \operatorname{Pr}\left[\mathcal{A}(x, \sigma, \pi)=1: \begin{array}{c}
(\sigma, \tau) \leftarrow S_{1}\left(1^{\lambda}, x\right) \\
\pi \leftarrow S_{2}(\sigma, \tau, x)
\end{array}\right] .
$$

Statistical Simulation-Soundness (SSS). A proof system is said to be statistically simulation sound if it is infeasible to convince an honest verifier of a false statement even when the adversary itself is provided with a simulated proof. For all statements $x$ and all (even unbounded) adversaries $\mathcal{A}$ we have

$$
\operatorname{Pr}\left[(\sigma, \tau) \leftarrow S_{1}\left(1^{\lambda}, x\right) ; \pi \leftarrow S_{2}(\sigma, \tau, x): \exists\left(x^{\prime}, \pi^{\prime}\right): x^{\prime} \neq x: V\left(\sigma, x^{\prime}, \pi^{\prime}\right)=1: x^{\prime} \notin L\right]=\operatorname{negl}(\lambda) .
$$

\section{B Overview of One-Time GRAM and Bitwise Compactness}

We briefly describe a high-level framework for construction one-time garbled RAM as defined in $\left[\mathrm{LO} 3 \mathrm{a}, \mathrm{GHL}^{+} 14\right]$. The original instantiation of [LO13a] had a subtle bug which was later fixed by $\left[\mathrm{GHL}^{+} 14\right]$. See $\left[\mathrm{GHL}^{+} 14\right]$ for full details. Our main focus here is on the syntax of the construction and explaining why it satisfies our efficiency requirements such as bit-wise compactness. We begin by recalling the RAM model of computation.

\section{B.1 Random Access Machines}

A RAM program can be represented by a CPU-Step Circuit

$$
\operatorname{CPU}\left(s, b^{\text {read }}\right)=\left(s^{\prime}, i^{\text {read }}, i^{\text {write }}, b^{\text {write }}\right),
$$

which also has access to external memory. The RAM program is executed by repeatedly taking as input the current state $s$ and data $b^{\text {read }}$ and applying the CPU-step function from above. This step determines the state for the next step $s^{\prime}$, some data $b^{\text {write }}$ to write to memory location $i$ write, and another memory location $i^{\text {read }}$ from which to read the data for the next step. The initial $s$ of the computation consists of the input, and the computation concludes when it reaches a designated "halt" $s$, at which point the output is found in the external memory, starting from position $i^{\text {read }}$. The initial memory content is often taken to be empty, but when we consider repeated computations with persistent memory then the memory content is assumed to persist from the previous execution.

\section{B.2 The One-Time GRAM Construction}

The main idea for how to garble a RAM program $P$ is to just use Yao garbled circuits to garble $t$ copies of the CPU step circuit as described above. The state of the computation $s$ remains 
garbled from one circuit to the next. The main difficulty is how to allow the computation to access (read/write) values in memory. To do so, we define a "garbled memory" which contains a secret keys $\mathrm{sk}_{i, j, b}$ for each memory location $i$, where $b$ corresponds to the bit that should be in that location and $j$ corresponds to the CPU step in which that bit was written (or 0 if it was initialized that way). Each garbled CPU step circuit also outputs (in the clear) the locations $i^{\text {read }}, i^{\text {write }}$ that it wants to read/write as well as:

- A secret key sk ${ }^{\text {write }}=\mathrm{sk}_{i \text { write }, j, b \text { write }}$ where $b^{\text {write }}$ is the bit being written to in location $i^{\text {write }}$ and $j$ is the current CPU step index.

- Two ciphertexts $c_{0}, c_{1}$ that encrypt the labels corresponding to bit 0 and 1 respectively for the wire corresponding to the bit $b^{\text {read }}$ in the next garbled circuit. The encryptions are created in such a way that $c_{b}$ can only be decrypted by $\mathrm{sk}_{i, j, b}$ where $j$ is the time period in which location $i$ was last written to.

Originally, the garbled memory consists of secret keys $\mathbf{s k}_{i, 0, D[i]}$ where $D[i]$ is the $i$ 'th bit in the persistent data $D$, or we set $D[i]=0$ in the case where we have no persistent data and just want to initialize the memory to all 0s. The evaluator starts evaluating the garbled CPU step circuits one-at-a-time. After each evaluation, it gets our the values $i^{\text {read }}, i^{\text {write }}$, sk $^{\text {write }}, c_{0}, c_{1}$. It writes sk ${ }^{\text {write }}$ to the location $i^{\text {write }}$ of garbled memory and reads $s k^{\text {read }}$ from location $i^{\text {read }}$. It attempts to decrypt both ciphertexts $c_{0}, c_{1}$ with $\mathrm{sk}^{\text {read }}$ and, depending on whichever one decrypts correctly, it uses the corresponding decrypted message as the label of the bit $b^{\text {read }}$ for the next garbled circuit.

Loose Ends. The above already described the main idea, but there are several loose ends in the above description:

- The evaluator learns all of the locations $i^{\text {read }}, i^{\text {write }}$ being accessed by the program, which can reveal sensitive information about the program execition. This can be fixed by using an oblivious RAM scheme to compile the computation into one where these locations do not reveal any private information.

- Each garbled CPU circuit, when reading location $i^{\text {read }}$ needs to know the index $j$ in which that location was last written to. There are generic ways to convert any program execution into one that makes this easy, and some ORAM constructions already have this type of "predictably-timed writes" property.

The original scheme from [LO13a] has one master secret key that was hard-coded in each garbled CPU step circuit and could be used to generate the values $c_{0}, c_{1}$, sk ${ }^{\text {write }}$. This lead to a subtle circularity problem as outlined in $\left[\mathrm{GHL}^{+} 14\right]$, but this can be fixed using identity-based encryption, as shown in $\left[\mathrm{GHL}^{+} 14\right.$, GHRW14]. Note that $\left[\mathrm{GHL}^{+} 14\right.$, LO14] also present an alternate approach which does not require identity-based encryption but loses in efficiency, and therefore we do not consider it in this work.

Efficiency. The main take-away from the above description is the following: to create a garbled one-time RAM program with run-time $t$, we need to create $t$ garbled copies of (an augmented version of) the CPU step circuit under Yao's garbled circuit construction. Each such circuit is of some small size poly $(\lambda)$. The circuits aren't completely independent - the labels of the output wires in one circuit must match the labels of the input wires of the next circuit. However, in Yao's garbled circuits, we can choose the wire labels pseduorandomly via a PRF of the wire identifier. Therefore, we can compute each of the $t$ garbled circuits individually in time poly $(\lambda)$ without computing all of the other circuits. In particular, there is a short circuit of size poly $(\lambda)$ that outputs the $i$ th bit 
of the one-time garbled program $\tilde{P}$ by only computing a single garbled circuit and outputting 1 bit of it.

\section{Reusable Garbled Circuits with Output-Size Independence}

We show that any construction of reusable garbled circuits satisfying simulation-based security must have have garbled inputs of length at least as large as the outputs of the circuit being garbled. In other words, if we garble a circuit with short inputs and huge outputs, the length of the garbled input will unfortunately need to be huge. Note that this is in contrast to standard (non-reusable) garbled circuits, for which the above does not hold. In our work, we also show that the above does not hold for reusable garbled circuits if we give up on simulation security, and instead only consider an indistinguishability-based security. The impossibility argument follows the "incompressibility analysis" used in [AGVW13]. On a high level, a simulator that can simulate many garbled inputs of a reusable garbled circuit given many outputs has found a way to "compress" the outputs: given the garbled inputs and the garbled circuit one can recover all the outputs. If the outputs are pseduorandom (e.g., the outputs of a pseudoranom generator) then this should be impossible.

Theorem C.1. Let GC = (GC.circ, GC.inp, GC.eval) be any reusable garbled-circuit scheme with simulation security. Then for any polynomial $m=m(\lambda)$ there is a family of circuits $C_{\lambda}: \lambda \rightarrow$ $m(\lambda)$ for which the garbled inputs must be of length at least $m$ : i.e., for $x \in\{0,1\}^{\lambda},(\tilde{C}, k) \leftarrow$ $\mathrm{GC} \cdot \operatorname{circ}\left(1^{\lambda}, C_{\lambda}\right), \tilde{x} \leftarrow \mathrm{GC} \cdot \operatorname{inp}(x, k)$, we have $|\tilde{x}| \geq m . \quad 14$

Proof. For any polynomial $m=m(\lambda)$, let prg : $\{0,1\}^{\lambda} \rightarrow\{0,1\}^{m}$ be a pseudo-random generator (PRG), let $C_{\mathrm{prg}, \lambda}$ be a circuit family computing the function prg. Assume that the theorem does not hold so there is some $\ell=\ell(\lambda)<m$ such that the garbled inputs to the garbled $C_{\mathrm{prg}, \lambda}$ are of size $\ell$. Let $p=p(\lambda)$ be some bound on the size of $\tilde{C}_{\mathrm{prg}, \lambda}$ given by $\left(\tilde{C}_{\mathrm{prg}, \lambda}, k\right) \leftarrow \mathrm{GC} \cdot \operatorname{circ}\left(1^{\lambda}, C_{\mathrm{prg}, \lambda}\right)$. Let $q=q(\lambda)$ be such that $q \cdot m>q \cdot \ell+p+\lambda$. Let Sim be the simulator for GC.

We construct a distinguisher Dist for the PRG. The distinguisher gets $q$ values $y_{1}, \ldots, y_{q}$ which are either all random or all pseudo-random. $\operatorname{Dist}\left(1^{\lambda}, y_{1}, \ldots, y_{q}\right)$ runs $\left(\tilde{C}_{\operatorname{prg}, \lambda}^{\prime}, \tilde{x}_{1}^{\prime}, \ldots, \tilde{x}_{q}^{\prime}\right) \leftarrow$ $\operatorname{Sim}\left(1^{\lambda}, C_{\mathrm{prg}}, y_{1}, \ldots, y_{q}\right)$. It tests if GC.eval $\left(\tilde{C}_{\mathrm{prg}, \lambda}^{\prime}, \tilde{x}_{i}^{\prime}\right)=y_{i}$ for all $i \in[q]$ and, if so, it outputs 1 else it outputs 0 .

We claim that if $y_{1}, \ldots, y_{q}$ are pseudorandom with $y_{i}=\operatorname{prg}\left(x_{i}\right)$ for some $x_{i} \in\{0,1\}^{\lambda}$, then $\operatorname{Dist}\left(y_{1}, \ldots, y_{q}\right)=1$ with probability $1-\operatorname{negl}(\lambda)$. This is because, by the security of the simulation, we have

$$
\left(\tilde{C}_{\mathrm{prg}, \lambda}^{\prime}, \tilde{x}_{1}^{\prime}, \ldots, \tilde{x}_{q}^{\prime}\right) \stackrel{\text { comp }}{\approx}\left(\tilde{C}_{\mathrm{prg}, \lambda}, \tilde{x}_{1}, \ldots, \tilde{x}_{q}\right)
$$

where $\left(\tilde{C}_{\mathrm{prg}, \lambda}, k\right) \leftarrow \mathrm{GC} \cdot \operatorname{circ}\left(1^{\lambda}, C_{\mathrm{prg}, \lambda}\right)$ is the real garbled circuit and $\tilde{x}_{i} \leftarrow \mathrm{GC} \cdot \operatorname{inp}\left(x_{i}, k\right)$ are the real garbled inputs. By the correctness of the garbled circuit we must have GC.eval $\left(\tilde{C}_{\mathrm{prg}, \lambda}, \tilde{x}_{i}\right)=y_{i}$ and therefore we must also have GC.eval $\left(\tilde{C}_{\mathrm{prg}, \lambda}^{\prime}, \tilde{x}_{i}^{\prime}\right)=y_{i}$ with overwhelming probability.

On the other hand, if $\bar{y}=\left(y_{1}, \ldots, y_{q}\right)$ is random then Dist $\left(y_{1}, \ldots, y_{q}\right)=1$ with probability $\operatorname{negl}(\lambda)$. This is because there are $2^{m q}$ possible values of $\bar{y}$ but only $2^{p+q \ell}$ possible values of $\left(\tilde{C}_{\mathrm{prg}, \lambda}^{\prime}, \tilde{x}_{1}^{\prime}, \ldots, \tilde{x}_{q}^{\prime}\right)$ of the right size. Therefore there is only a $2^{m q-(p+q \ell)}=2^{-\lambda}$ fraction of values $\bar{y}$ that the simulator can "explain" with some $\left(\tilde{C}_{\mathrm{prg}, \lambda}^{\prime}, \tilde{x}_{1}^{\prime}, \ldots, \tilde{x}_{q}^{\prime}\right)$.

Together, this proves the theorem.

\footnotetext{
${ }^{14}$ For simplicity, we assume that for a fixed $C_{\lambda}$, the sizes of $\tilde{C}, \tilde{x}$ are also fixed and do not vary.
} 


\section{Functional Encryption for Random Access Machines}

In this section we present a construction of a functional encryption (FE) scheme for RAM programs using functional encryption for circuits as a building block. In both cases, we consider indistinguishability-based security, and it is known that simulation security is unachievable in this setting when an attacker can get keys for arbitrarily many different programs/functions [AGVW13]. However, we then show a simple transformation from functional encryption for RAMs that satisfies indistinguishability-based security into reusable garbled RAM with simulation security. Therefore, this section gives us an alternate approach to the one in Section 3.3 for constructing reusable garbled RAM (without persistent memory) from indistinguishability-based functional encryption for circuits.

\section{D.1 Definitions}

First, we present a formal definition for FE for RAMs and the indistinguishability security notion for it.

Definition D.1 (Functional Encryption for RAMs). A functional encryption scheme for RAM programs consists of four algorithms FEram $=$ (FEram.setup, FEram.keygen, FEram.enc, FEram.dec):

- $(\mathrm{PK}, \mathrm{MSK}) \leftarrow$ FEram.setup $\left(1^{\lambda}\right)$ - takes as input the security parameter $\lambda$ and outputs a public parameters PK and a master secret key MSK.

- $\mathrm{SK}_{P} \leftarrow$ FEram.keygen(MSK, $\left.P,(n, t, m)\right)$ - a polynomial time algorithm that takes as input the master secret key MSK and a description of a RAM program $P$ with input size $n$, output size $m$, and run-time bound $t$, and outputs a corresponding secret key $\mathrm{SK}_{P}$.

- $c \leftarrow$ FEram.enc(PK, $x)$ - a polynomial time algorithm that takes the public parameters PK and a string $x \in\{0,1\}^{n}$ and outputs a ciphertext $c$.

- $y \leftarrow$ FEram.dec $\left(\mathrm{SK}_{P}, c\right)$ - a polynomial time algorithm that takes a secret key $S K_{P}$ and ciphertext encrypting message $x \in\{0,1\}^{n}$ and outputs $P(m)$.

A functional encryption scheme is correct for all RAM programs $P$ with run-time $t$, input size $n$, and output size $m$, and all messages $x \in\{0,1\}^{n}$, all (PK, MSK $) \leftarrow$ FEram.setup $\left(1^{\lambda}\right), \mathrm{SK}_{P} \leftarrow$ FEram.keygen(MSK, $P,(n, t, m))$, all $c \leftarrow$ FEram.enc $(\mathrm{PK}, x)$, we have FEram.dec $\left(\mathrm{SK}_{P}, c\right)=P(x)$.

Indistinguishability Security. We now define indistinguishability security for functional encryption. Intuitively, this notion says that the attacker cannot distinguish the encryptions of two different messages $x_{0}, x_{1}$ even given secret keys $\mathrm{SK}_{P}$ for various programs $P$ as long as $P\left(x_{0}\right)=P\left(x_{1}\right)$. We will only consider selective security where the attacker picks the challenge messages ahead of time before seeing PK. (Note that selective security automatically implies fully adaptive security via a reduction that simply guesses the message and therefore loses a factor of $2^{-n}$ in advantage, where $n$ is the message size.) For simplicity, we will make selective security our default notion and avoid specifying this in the future. We consider the following game between an attacker $\mathcal{A}$ and a challenger.

Challenge: $\mathcal{A}$ submits two messages $x_{0}, x_{1} \in\{0,1\} n$.

Setup: The challenger runs (PK, MSK) $\leftarrow$ FEram.setup $\left(1^{\lambda}\right)$, picks a bit $b \leftarrow\{0,1\}$ at random, and computes $c \leftarrow$ FEram.enc(PK, $\left.x_{b}\right)$. It gives PK, $c$ to $\mathcal{A}$. 
Key Query: $\mathcal{A}$ adaptively submits queries consisting of $P, t, m$ and is given

$\mathrm{SK}_{P} \leftarrow$ FEram.keygen(MSK, $\left.P,(n, t, m)\right)$. This step can be repeated any polynomial number of times by the attacker.

Guess: $\mathcal{A}$ eventually outputs a bit $b^{\prime} \in\{0,1\}$.

We say that $\mathcal{A}$ is legal if the messages $x_{0}, x_{1}$ and the key queries $\left(P_{i}, t_{i}, m_{i}\right)$ satisfy $P_{i}\left(x_{0}\right)=P_{i}\left(x_{1}\right)$ for all $i$, where both executions finish in at most $t_{i}$ steps. The advantage of an algorithm $\mathcal{A}$ in this game is $\operatorname{Adv}_{\mathcal{A}}=\operatorname{Pr}\left[b^{\prime}=b\right]-\frac{1}{2}$.

Definition D.2. An functional encryption scheme for $R A M$ programs has indistinguishability security if for all poly-time legal attackers $\mathcal{A}$ the function $\operatorname{Adv}_{\mathcal{A}}(\lambda)$ is negligible.

We also define q-key-query indistinguishability security, where the attacker $\mathcal{A}$ is further restricted to making at most q key queries.

Functional Encryption for Circuits. The notion of functional encryption for circuits satisfying indistinguishability security was recently defined and constructed by [GGH $\left.{ }^{+} 13 \mathrm{~b}\right]$ using indistinguishability obfuscation. We will show how to transform functional encryption for circuits into one for RAMs in a black-box way. A functional encryption scheme for circuits consists of algorithms FEcirc $=($ FEcirc.setup, FEcirc.keygen, FEcirc.enc, FEcirc.dec) which have essentially the same syntax as the RAM scheme, except that the algorithm $\mathrm{SK}_{C} \leftarrow$ FEcirc.keygen(MSK, $C$ ) now takes as input some circuit $C$ with input size $n$ and arbitrary output size $m .{ }^{15}$ The indistinguishability security game is the same for circuits as for RAM schemes, with the obvious syntactical modifications.

\section{D.2 Our Construction}

We rely on similar techniques to the ones needed to construct reusable garbled RAM without persistent memory from Section 3.3 to construct functional encryption from RAMs. The overall idea for our construction will combine a functional encryption scheme for circuits with one-time garbled RAM. More specifically the decryption key for an FE scheme for RAMs will be an FE decryption key for a circuit that outputs one time use garbled RAM program and the corresponding garbled input for it. For the following, let FEcirc $=($ FEcirc.setup, FEcirc.keygen, FEcirc.enc, FEcirc.dec $)$ be a functional encryption scheme for circuits and let GR1 = (GR1.prog, GR1.inp, GR1.eval) be a one-time garbled-RAM scheme.

The RAM-Garbling Circuits. We first show how to translate a RAM program $P$ for which we want to create an $\mathrm{FE}$ secret key into a corresponding circuit $C_{F E}[P, \ldots]$ on which we will call the circuit $\mathrm{FE}$ scheme. Let $F_{K}(\cdot)$ be a pseudorandom function with key size $|K|=\lambda$, input size $\lambda$ and variable-length output size. Let $P$ be a RAM program with input size $n$, output size $m$ and run-time $t$. Assume that for these parameters, the one-time garbled RAM scheme $(\tilde{P}, s) \leftarrow \mathrm{GR} 1 \cdot \operatorname{prog}\left(1^{\lambda}, P,(n, m, t) ; r_{1}\right), \tilde{x} \leftarrow \mathrm{GR} 1 \cdot \operatorname{inp}\left(x, s,(n, m, t) ; r_{2}\right)$, has output size $|\tilde{P}, \tilde{x}|=M$ and randomness sizes $\left|r_{1}\right|=\ell_{1},\left|r_{2}\right|=\ell_{2}$.

\footnotetext{
${ }^{15}$ Alternatively, we could restrict ourselves to circuits with 1-bit output. In the case of unbounded key queries, the two notions are equivalent since we can always just give a separate key for each output bit of the circuit.
} 


$\frac{\mathbf{C}_{\mathbf{F E}}[\mathbf{P}, \mathbf{n}, \mathbf{m}, \mathbf{t}, \mathbf{V}, \text { id }, \lambda]\left(x, K_{0}, K_{1}, \text { flag }\right):}{/ / K_{0}, K_{1}, \text { id } \in\{0,1\}^{\lambda}, x \in\{0,1\}^{n}, V \in\{0,1\}^{M}, \text { flag } \in\{0,1\}}$
If flag $=1$, compute $R=F_{K_{1}}($ id $)$ of size $|R|=M$ and output $R \oplus V$.
Else if flag $=0$ :
$\quad$ 1. Generate $\left(r_{1}, r_{2}\right) \leftarrow F_{K_{0}}($ id $)$ of size $\left|r_{1}\right|=\ell_{1},\left|r_{2}\right|=\ell_{2}$.
$\quad$ 2. Run $(\tilde{P}, s) \leftarrow$ GR $1 . \operatorname{prog}\left(1^{\lambda}, P,(n, m, t) ; r_{1}\right), \tilde{x} \leftarrow \operatorname{GR} 1 . \operatorname{inp}\left(x, s,(n, m, t) ; r_{2}\right)$,
$\quad$ 3. Output $(\tilde{P}, \tilde{x})$.

Functional Encryption for RAMs. We describe our FE for RAMs construction FEram = (FEram.setup, FEram.keygen, FEram.enc, FEram.dec) in the following figure.

FEram.setup $\left(1^{\lambda}\right)$ :

1. Run $(\mathrm{PK}, \mathrm{MSK}) \leftarrow$ FEcirc.setup $\left(1^{\lambda}\right)$.

2. Output (PK, MSK).

FEram.keygen(MSK, $P,(n, t, m))$ :

1. Choose a random string $V \leftarrow\{0,1\}^{M}$ and a random identifier id $\leftarrow\{0,1\}^{\lambda}$.

2. Construct the circuit $C_{F E}=C_{F E}[P, n, m, t, V$, id, $\lambda]$ as shown above.

3. Output $\mathrm{SK}_{C_{F E}} \leftarrow$ FEcirc.keygen(MSK, $C_{F E}$ ).

FEram.enc $(\mathrm{PK}, x)$ :

1. Choose a random $K_{0} \leftarrow\{0,1\}^{\lambda}$, set $w=\left(x, K_{0}, 0^{\lambda}, 0\right)$.

2. Output the encryption $c \leftarrow$ FEcirc.enc $(\mathrm{PK}, w)$.

FEram.dec $\left(\mathrm{SK}_{C_{F E}[P, \ldots]}, c\right)$ :

1. Run the FE decryption $(\tilde{P}, \tilde{x}) \leftarrow$ FEcirc.dec $\left(\mathrm{SK}_{C_{F E}[P, \ldots]}, c\right) . \quad / /=C_{F E}[P, n, m, t, V, \mathrm{id}, \lambda](w)$

2. Evaluate the 1-time GRAM and output $y \leftarrow \operatorname{GR} 1 . \operatorname{eval}(\tilde{P}, \tilde{x}) . / /=P(x)$

Theorem D.3. If FEcirc $=$ (FEcirc.setup, FEcirc.keygen, FEcirc.enc, FEcirc.dec) is a functional encryption scheme satisfying indistinguishability security, and GR1 = (GR1.prog, GR1.inp, GR1.eval) is a one-time garbled-RAM scheme, then the scheme FEram above is a functional encryption scheme satisfying indistinguishability security. Moreover, if FEcirc only satisfies q-key-query indistinguishability security for some bounded $q$, then so does FEram.

Proof. We prove the theorem via a sequence of hybrid arguments.

- Hyb $\mathrm{b}_{0}$ : Let this be the indistinguishability security game with the challenger using the bit $b=0$. In particular, the challenger computes

$$
\begin{gathered}
c \leftarrow \operatorname{FEram} . \operatorname{enc}\left(x_{0}\right)\left\{\operatorname{FEcirc.enc}\left(\mathrm{PK},\left(x_{0}, K_{0}, \overline{0}, \text { flag }:=0\right)\right)\right\} \\
\left.\mathrm{SK}_{P_{i}} \leftarrow \text { FEram.keygen }\left(P_{i},(n, t, m)\right)\left\{\text { FEcirc.keygen(MSK, } C_{F E}[P, n, m, t, V, \text { id, } \lambda]\right)\right\}
\end{gathered}
$$

where $K_{0} \leftarrow\{0,1\}^{\lambda}$, id $\leftarrow\{0,1\}^{\lambda}, V \leftarrow\{0,1\}^{M}$.

- $\mathrm{Hyb}_{1}$ : in this hybrid, we change the way the decryption keys $\mathrm{SK}_{P}$ are generated for each key query $(P, n, t, m)$. At the very beginning of the game, we choose a random PRF key $K_{1} \leftarrow\{0,1\}^{\lambda}$. To compute $\mathrm{SK}_{P}$ we do the following: 
- Choose a random id $\leftarrow\{0,1\}^{\lambda}$ as before.

- Compute $\left(r_{0}, r_{1}\right)=F_{K_{0}}($ id $),(\tilde{P}, s) \leftarrow \mathrm{GR} 1 \cdot \operatorname{prog}\left(1^{\lambda}, P,(n, m, t) ; r_{1}\right)$, $\tilde{x} \leftarrow \operatorname{GR} 1 \cdot \operatorname{inp}\left(x_{0}, s,(n, m, t) ; r_{2}\right)$.

- Set $V:=(\tilde{P}, \tilde{x}) \oplus F_{K_{1}}$ (id).

- Output as decryption key $\mathrm{SK}_{P} \leftarrow$ FEcirc.keygen(MSK, $C_{F E}[P, n, m, t, V$, id, $\left.\lambda]\right)$.

Notice that the only difference between $\mathrm{Hyb}_{0}$ and $\mathrm{Hyb}_{1}$ is the way that $V$ is generated. Previously, it was uniformly random for each key, whereas now it is computed as $V:=$ $(\tilde{P}, \tilde{x}) \oplus F_{K_{1}^{\prime}}($ id $)$.

The indistinguishability of $\mathrm{Hyb}_{0}$ and $\mathrm{Hyb}_{1}$ follows from the pseudorandom property of $F$ under key $K_{1}$ (which does not appear anywhere else in the game, and in particular, is not used during the creation of the ciphertext $c$ ). We also rely on the fact that id is chosen randomly in each key query and therefore is fresh with overwhelming probability.

- $\mathrm{Hyb}_{2}$ : in this hybrid we set the challenge ciphertext as FEcirc.enc(PK, $\left(0, \overline{0}, K_{1}\right.$, flag $\left.=1\right)$ ).

The indistinguishability of $\mathrm{Hyb}_{1}$ and $\mathrm{Hyb}_{2}$ follows from the $\mathrm{FE}$ indistinguishability security of FEcirc since, for all of the queried programs $P$, we have

$$
C_{F E}[P, n, m, t, V \text {, id, } \lambda]\left(x_{0}, K_{0}, \overline{0}, 0\right)=C_{F E}[P, n, m, t, V \text {, id, } \lambda]\left(0, \overline{0}, K_{1}, 1\right)=(\tilde{P}, \tilde{x})
$$

where $\left(r_{0}, r_{1}\right)=F_{K_{0}}$ (id), $(\tilde{P}, s) \leftarrow \mathrm{GR} 1 \cdot \operatorname{prog}\left(1^{\lambda}, P,(n, m, t) ; r_{1}\right), \tilde{x} \leftarrow \mathrm{GR} 1 \cdot \operatorname{inp}\left(x_{0}, s,(n, m, t) ; r_{2}\right)$.

Furthermore, note that if we only have $q$ key-queries in the RAM FE security game, then we only need $q$ key-queries for the circuit FE security to prove indistinguishability of these hybrids.

- $\mathrm{Hyb}_{3}$ : In this hybrid we change how the value $V$ is chosen in each key query when constructing the circuit $C_{F E}[P, n, m, t, V$, id, $\lambda]$. In particular, instead of choosing $\left(r_{0}, r_{1}\right)=F_{K_{0}}$ (id) pseudorandomly, we choose $\left(r_{0}, r_{1}\right)$ uniformly at random. Then we continue as before to compute $(\tilde{P}, s) \leftarrow \mathrm{GR} 1 . \operatorname{prog}\left(1^{\lambda}, P,(n, m, t) ; r_{1}\right), \tilde{x} \leftarrow \mathrm{GR} 1 . \operatorname{inp}\left(x_{0}, s,(n, m, t) ; r_{2}\right)$ and set $V:=$ $(\tilde{P}, \tilde{x}) \oplus F_{K_{1}}($ id $)$.

The indistinguishability of $\mathrm{Hyb}_{2}$ and $\mathrm{Hyb}_{3}$ follows from the pseudorandom property of $F$ under key $K_{0}$, which is not used anywhere else in either game beyond computing the values $V$.

- $\mathrm{Hyb}_{4}$ : In this hybrid we change how the value $V$ is chosen in each key query when constructing the circuit $C_{F E}[P, n, m, t, V$, id, $\lambda]$ once again. In particular, after choosing $(\tilde{P}, s) \leftarrow$ GR1.prog $\left(1^{\lambda}, P,(n, m, t) ; r_{1}\right)$, instead of setting $\tilde{x} \leftarrow \mathrm{GR} 1 . \operatorname{inp}\left(x_{0}, s,(n, m, t) ; r_{2}\right)$ to be a garbling of $x_{0}$, we now set it to $\tilde{x} \leftarrow \operatorname{GR} 1 . \operatorname{inp}\left(x_{1}, s,(n, m, t) ; r_{2}\right)$ to be a garbling of $x_{1}$. Then we again set $V:=(\tilde{P}, \tilde{x}) \oplus F_{K_{1}}$ (id).

The indistinguishability of $\mathrm{Hyb}_{3}$ and $\mathrm{Hyb}_{4}$ follows from the one-time security of the garbled RAM scheme and the fact that $P\left(x_{0}\right)=P\left(x_{1}\right)$ for each program $P$. In particular, for each program $P$ we have:

$$
\left(\tilde{P}, \tilde{x}_{0}\right) \stackrel{\operatorname{comp}}{\approx} \operatorname{GR} 1 . \operatorname{Sim}\left(1^{\lambda}, P,(n, m, t), P\left(x_{0}\right)=P\left(x_{1}\right)\right) \stackrel{\text { comp }}{\approx}\left(\tilde{P}, \tilde{x}_{1}\right)
$$

where $(\tilde{P}, s) \leftarrow \mathrm{GR} 1 \cdot \operatorname{prog}\left(1^{\lambda}, P,(n, m, t) ; r_{1}\right), \tilde{x}_{e} \leftarrow \mathrm{GR} 1 \cdot \operatorname{inp}\left(x_{e}, s,(n, m, t) ; r_{2}\right)$, for $e \in\{0,1\}$. 
- $\mathrm{Hyb}_{5}-\mathrm{Hyb}_{7}$ : We now do everything in reverse. In particular, hybrids 5,6,7 are the same as hybrids $3,2,1$ (respectively) with the only difference being that $x_{1}$ is used instead of $x_{0}$.

In particular, in hybrid 5 we again choose $\left(r_{0}, r_{1}\right)=F_{K_{0}}$ (id) pseudorandomly instead of randomly (security of PRF with $K_{0}$ ). In hybrid 6, we compute the challenge ciphertext honestly as FEcirc.enc(PK, $\left(x_{1}, K_{0}, \overline{0}\right.$, flag $\left.=0\right)$ ) (FE security for circuits). In hybrid 7 we go back to choosing $V \leftarrow\{0,1\}^{M}$ uniformly at random (security of PRF with $K_{1}$ ).

Notice that Hybrid 7 is the FE RAM indistinguishability game with the challenge bit $b=1$. Therefore, this proves the theorem.

\section{D.3 Efficiency Analysis}

The efficiency of the setup procedure is the same for FEram scheme as for the underlying FEcirc scheme, which is poly $(\lambda)$. Similarly the efficiency of encryption is the same for FEram and FEcirc, up to an $O(\lambda)$ additive overhead in the message size $n$. We assume that the complexity is $n \cdot \operatorname{poly}(\lambda)$.

For a program $P$ with run-time bound $t$, input size $n$ and output size $m$, the efficiency of key generation and decryption of the FE-RAM scheme are the same as those of the underlying FECircuit scheme with the circuit $C_{F E}=C_{F E}[P, \ldots]$. Recalling the parameters of one-time GRAM in Section 5, the size of the circuit $C_{F E}$ is $q=\widetilde{O}(t+|P|+n+m) \cdot \operatorname{poly}(\lambda)$, its output size is $m^{\prime}=\widetilde{O}(t+|P|+n+m) \cdot \operatorname{poly}(\lambda)$ and its input size is $n^{\prime}=n+O(\lambda)$. Moreover, the circuit $C_{F E}$ is $s$-weakly bitwise compact (see Definition 5.1) where $s=\widetilde{O}(|P|+n) \cdot \operatorname{poly}(\lambda, \log t)$.

If we do not make any assumptions on the efficiency of the underlying FEcirc scheme and use it naively, then the efficiency of key generation and decryption could be poly $(q, \lambda)=\operatorname{poly}(t)$ which could potentially obliterate all efficiency benefits of using a RAM rather than converting it into a circuit. However, we can leverage bitwise compactness to recover efficiency by assuming that the FEcirc scheme is optimized, similarly to the analysis in Section 5. In particular, for $i=1, \ldots, m^{\prime}$, let $C_{b i t, i}^{F E}$ be the bitwise representation circuits for $C_{F E}$.

- Optimized Circuit FE: An optimized FE scheme for circuits works as follows. Given a bitwise compact circuit $C_{F E}$, the key-generation procedure $\mathrm{SK}_{C_{F E}} \leftarrow$ FEcirc.keygen(MSK, $C^{F E}$ ) computes $\mathrm{SK}_{C_{F E}}=\left(\mathrm{SK}_{1}, \ldots, \mathrm{SK}_{m^{\prime}}\right)$ where $\mathrm{SK}_{i} \leftarrow$ FEcirc.keygen $\left(\mathrm{MSK}, C_{b i t, i}^{F E}\right)$. The decryption procedure computes FEcirc.dec $\left(\mathrm{SK}_{C_{F E}}, c\right)=\left(b_{1}, \ldots, b_{m^{\prime}}\right)$ where $b_{i}:=$ FEcirc.dec $\left(\mathrm{SK}_{i}, c\right)$.

In this case the efficiency of key generation and decryption is $\widetilde{O}(t) \cdot \operatorname{poly}(|P|+n+\lambda)$. Note that the security of the optimized scheme follows directly from the security of the underlying scheme, and therefore we can assume that the scheme is optimization without loss of generality.

\section{D.4 From FE for RAMs to Reusable Garbled RAM}

We note that indistignuishability-secure FE for RAMs with unlimited key queries can also be converted into a simulation-secure FE for RAMs with 1 key query. This is done via the same "real-or-dummy" program paradigm we used in Section 3.3 and based on the ideas of De Caro et al. [CIJ ${ }^{+}$13] which showed how to convert indistinguishability-based security to simulation-based security for functional encryption for circuits. The notion of "simulation-secure FE for RAMs with 1 key query" is essentially the same as reusable garbled RAM with public input garbling. Therefore we present the result in that terminology.

For a program $P(x)$, let $P^{+}(x, \psi, y)$ be the real-or-dummy program define in Section 3.3 which outputs $P(x)$ if $\psi=0$ (real) or $y$ if $\psi=1$ (dummy). Let FEram $=$ (FEram.setup, FEram.keygen, FEram.enc, FEram.dec) be a functional encryption scheme satisfying indistinguishability security with $q=1$ key queries. We define GR = (GR.prog, GR.inp, GR.eval) via: 
- GR.prog $(P,(n, m, t)):$ Compute $(\mathrm{MPK}, \mathrm{MSK}) \leftarrow$ FEram.setup $\left(1^{\lambda}\right)$,

$\tilde{P} \leftarrow$ FEram.keygen(MSK, $\left.P^{+},(n+m+1, t, m)\right)$. Output $(\tilde{P}, s=\mathrm{MPK})$.

- $\operatorname{GR} \cdot \operatorname{inp}(x, s=\operatorname{MPK},(n, m, t))$ : Return $\tilde{x} \leftarrow$ FEram.enc(MPK, $\left.\left(x, \psi=0,0^{m}\right)\right)$.

- $\mathrm{GR} \cdot \operatorname{eval}(\tilde{P}, \tilde{x}):$ Output FEram.dec $(\tilde{P}, \tilde{x})$.

Theorem D.4. If FEram satisfies indistinguishability based security for $q=1$ key queries, then GR defined above is a resuable garbled RAM scheme with public input garbling.

Proof. The correctness and efficiency requirements follow immediately.

For security, we define the simulator $\operatorname{Sim}\left(1^{\lambda}, P,(n, m, t), y_{1}, \ldots, y_{\ell}\right)$ generates $(\tilde{P}, s=\mathrm{MPK}) \leftarrow$ GR.prog $(P,(n, m, t))$ and $\tilde{x}_{i}^{\prime} \leftarrow$ FEram.enc(MPK, $\left.\left(0^{n}, \psi=1, y_{i}\right)\right)$ for $i=1, \ldots, \ell$.

To prove security we simply need to show that for every program $P$ with parameter $(n, m, t)$ and any $\ell$ inputs $x_{1}, \ldots, x_{\ell}$ such that $P\left(x_{i}\right)=y_{i}$ we have

$$
\left(\mathrm{MPK}, \tilde{P}, \tilde{x}_{1}, \ldots, \tilde{x}_{\ell}\right) \stackrel{\text { comp }}{\approx}\left(\mathrm{MPK}, \tilde{P}, \tilde{x}_{1}^{\prime}, \ldots, \tilde{x}_{\ell}^{\prime}\right)
$$

where $\tilde{x}_{i} \leftarrow \operatorname{GR} \cdot \operatorname{inp}\left(x_{i}, s\right):\left\{\right.$ FEram.enc $\left(M P K,\left(x_{i}, \psi=0,0^{m}\right)\right\}$. Since we have public input garbling, this simply follows via a hybrid argument as long as we show that for each $i \in[\ell]$ :

$$
\left(\mathrm{MPK}, \tilde{P}, \tilde{x}_{i}\right) \stackrel{\text { comp }}{\approx}\left(\mathrm{MPK}, \tilde{P}, \tilde{x}_{i}^{\prime}\right)
$$

Note that $P^{+}\left(x_{i}, \psi=0,0^{m}\right)=y_{i}=P^{+}\left(0^{n}, \psi=1, y_{i}\right)$. Therefore, the above follows directly from FE indistinguishability security for RAMs with a single key query for program $P^{+}$and challenge messages $w_{0}=\left(x_{i}, \psi=0,0^{m}\right), w_{1}=\left(0^{n}, \psi=1, y_{i}\right)$.

Using the efficiency of the functional encryption schemes from the literature $\left(\left[\mathrm{GGH}^{+} 13 \mathrm{~b}\right]\right)$ the above construction achieves the same asymptotic efficiency as that of Theorem 5.4.

\section{E Obfuscation for RAMs}

We propose a candidate construction of obfuscation for random access machines using obfuscation for circuits. In particular, such scheme ensures that the size of the obfuscated program and the running time of the obfuscated program are only proportional to the size and worst-case runningtime of the original program. We note that the recent works of $\left[\mathrm{BCP} 13, \mathrm{ABG}^{+} 13\right]$ construct obfuscation for Turing Machines (where the size and running-time of the obfuscated program are proportional to the TM size and running time) assuming differing-inputs obfuscation.

Our idea is similar to our construction of reusable garbled RAM without persistent memory. We simply generate an obfuscation for a circuit $C(x)$ that, on input $x$, will output a one-time GRAM $\tilde{P}$ of the program $P$ and a corresponding garbled input $\tilde{x}$. The randomness used to generate $\tilde{P}$ and $\tilde{x}$ is simply chosen by evaluating a pseudorandom function $F_{K}(x)$ on the input $x$, where the key $K$ is hard-coded in the circuit $C$. Unfortunately, the circuit $C$ itself is huge, but as noted in Section 5 , it is bit-wise compact. In particular, there is a small circuit $C_{b i t}(x, i)$ that outputs the $i$ 'th bit of $C(x)$. Therefore, we will simply obfuscate $C_{b i t}$.

The main differences between this approach and our constructions of reusable garbled RAM are that: (1) the input to the circuit is given in the clear rather than being encrypted, (2) the randomness used to create $\tilde{P}, \tilde{x}$ is created via a PRF of $x$ rather than being provided as part of the encrypted input. The security goals are also vastly different: in the case of reusable garbled RAM we are attempting to hide something about the encrypted input $x$ whereas here we are trying to hide something about the program $P$. One implication of this is that we will need to rely on a 
one-time GRAM scheme that satisfies program privacy. This means that we modify Definition 3.1 so that the simulator does not get the program $P$. As mentioned in Section 6 , there are standard techniques for adding program privacy.

Let $P$ be a program with input size $n$, output size $m$, and worst-case running time $t$. We define the following circuit for our obfuscation construction.

$\mathbf{C}[\mathbf{P}, \mathbf{n}, \mathbf{m}, \mathbf{t}, \lambda, \mathbf{K}](x): \quad / / x \in\{0,1\}^{n}$

1. Generate randomness for the execution $\left(r_{1}, r_{2}\right) \leftarrow F(K, x)$.

2. Run $(\tilde{P}, s) \leftarrow \mathrm{GR} 1 \cdot \operatorname{prog}\left(1^{\lambda}, P,(n, m, t) ; r_{1}\right)$. $\tilde{x} \leftarrow \mathrm{GR} 1 . \operatorname{inp}\left(x, s,(n, m, t) ; r_{2}\right)$,

3. Output $(\tilde{P}, \tilde{x})$.

We also define the circuit $C_{b i t}[P, n, m, t, \lambda, K](x, i)$ which gets as input $i$ and only outputs the $i$ 'th bit of the output of $C[P, n, m, t, \lambda, K](x)$. As noted in Section 5 , the latter can be expressed as a circuit of size $\widetilde{O}(|P|+n+m) \cdot \operatorname{poly}(\lambda, \log t)$ where $t$ is the worst-case running time of $P$.

We define an obfuscation scheme for RAMs $\mathcal{O}_{R A M}=$ (Obfuscate, Evaluate) as follows:

- Obfuscate $(P)$ : On input a RAM program $P$ with input and output size $n$ and $m$ and running time $t$, generate a PRF key $K$ and output an obfuscation $\tilde{C} \leftarrow \mathcal{O}\left(C_{b i t}[P, n, m, t, \lambda, K]\right)$.

- Evaluate $(\tilde{C}, x)$ : Evaluate $\tilde{C}(x, i)$ for sufficiently many output bits $i=1, \ldots$, so as to obtain a one-time use garbled program $\tilde{P}$ and a garble input for it $\tilde{x}$. Output $y \leftarrow \operatorname{GR} 1 . \operatorname{eval}(\tilde{P}, \tilde{x})$.

The above construction would give us a virtual black-box obfuscation for RAMs if we assume VBB obfuscation for circuits. We make the following conjecture.

Conjecture E.1. There exists a pseudorandom function $F$, a one-time use garbled-RAM scheme $\mathrm{GR} 1=$ (GR1.prog, GR1.inp, GR1.eval) and an obfuscation $\mathcal{O}$, for which the above construction $\mathcal{O}_{R A M}=$ (Obfuscate, Evaluate) is an indistinguishability obfuscation for RAMs.

We note that our conjecture does not formally require full VBB security from $\mathcal{O}$. None of the known negative results for obfuscation seem to apply to the above conjecture, when instantiated with "standard-constructions" of the underlying primitives and a "good" obfuscator $\mathcal{O}$ such as the candidate from $\left[\mathrm{GGH}^{+} 13 \mathrm{~b}\right]$.

\section{F Proofs Omitted from Main Body}

\section{F.1 Proof of Theorem 3.5}

Proof of Theorem 3.5. The simulator was sketched above: On input $\left(1^{\lambda},(n, m, t), P, y_{1}, \ldots, y_{q}\right)$ with $\left|y_{i}\right|=m$ for all $i$, the simulator GR.sim begins just as the garbling procedure of the actual scheme, namely by constructing the circuit $C[P, n, m, t, \lambda]$ and applying to it the circuit-garbling procedure to get $(\tilde{C}, s) \leftarrow \mathrm{GC} \cdot \operatorname{circ}\left(1^{\lambda}, C[P, n, m, t, \lambda]\right)$. Next, for every $y_{i}$ the simulator chooses a uniformly random $r_{i} \in\{0,1\}^{2 \lambda}$, sets $w_{i}^{\prime}=\left(r_{i}, 0, \psi=0, y_{i}\right)$ and $\tilde{w}_{i}^{\prime} \leftarrow \mathrm{GC} \cdot \operatorname{inp}\left(w_{i}, s\right)$. The output of the simulator GR.sim consists of $\left(\tilde{C}, \tilde{w}_{1}^{\prime}, \ldots, \tilde{w}_{q}^{\prime}\right)$.

We next prove indistinguishability between the real and simulated outputs. Fix the program $P$ and inputs $x_{1}, \ldots, x_{q}$ for $P$, and denote $y_{i}=P\left(x_{i}\right)$ for all $i$. Let $w_{i}=\left(r_{i}, x_{i}, \psi=1,0^{m}\right)$ where $r_{i} \in\{0,1\}^{2 \lambda}$. We first argue that the output distributions on inputs $w_{i}$ and $w_{i}^{\prime}$ are indistinguishable.

Claim F.1. Denote $C=C[P, n, m, t, \lambda]$. If the scheme GR1 = (GR1.prog, GR1.inp, GR1.eval) satisfies one-time GRAM security then for every $x \in\{0,1\}^{n}$ and $y=P(x)$ we have

$$
\left\{C\left(w_{i}\right)\right\} \stackrel{\operatorname{comp}}{\approx}\left\{C\left(w_{i}^{\prime}\right)\right\}
$$


where $w_{i}, w_{i}^{\prime}$ are chosen as described above.

Proof. Note that $C\left(w_{i}\right)=\left(\tilde{P}_{i}, \tilde{x}_{i}\right)$ and $C\left(w_{i}^{\prime}\right)=\left(\tilde{P}_{i}, \tilde{x}_{i}^{\prime}\right)$ where $\tilde{P}_{i}$ is a garbled version of the program $P^{+}, \tilde{x}_{i}$ is a garble version of the input $\left(x_{i}, 1,0^{m}\right)$ and $\tilde{x}_{i}^{\prime}$ is a garbled version of the input $\left(0^{n}, 0, y_{i}\right)$. The one-time simulation-security of the underlying GR1 scheme implies that:

$$
\left(\tilde{P}_{i}, \tilde{x}_{i}\right) \stackrel{\operatorname{comp}}{\approx} \operatorname{GR} 1 \cdot \operatorname{Sim}\left(1^{\lambda}, P,(n, m, t), y_{i}\right) \stackrel{\operatorname{comp}}{\approx}\left(\tilde{P}_{i}, \tilde{x}_{i}^{\prime}\right)
$$

since $y_{i}=P^{+}\left(\left(x_{i}, 1,0^{m}\right)\right)=P^{+}\left(\left(0^{n}, 0, y_{i}\right)\right)$. This proves the claim.

Claim F.1 implies that the distributions of the $w_{i}, w_{i}^{\prime}$ 's satisfy the condition of Definition 3.4, and by the distributional indistinguishability of GC we conclude that also

$$
\left\langle\tilde{C}, \tilde{w}_{1}, \ldots \tilde{w}_{q}\right\rangle \stackrel{\text { comp }}{\approx}\left\langle\tilde{C}, \tilde{w}_{1}^{\prime}, \ldots \tilde{w}_{q}^{\prime}\right\rangle .
$$

This completes the proof, since these are exactly the output distributions of the scheme GR and its simulator GR.sim.

\section{F.2 Proof of Theorem 3.6}

Proof of 3.6. Let $C=\left\{C_{\lambda}\right\}$ be a circuit (ensemble) and $D_{1}, \ldots, D_{q}$ and $D_{1}^{\prime}, \ldots, D_{q}^{\prime}$ be efficiently samplable input distributions (ensembles) such that, for all $j=1, \ldots, q$ it holds that

$$
\left\{C\left(x_{j}\right): x_{j} \leftarrow D_{j}\left(1^{\lambda}\right)\right\} \stackrel{\text { comp }}{\approx}\left\{C\left(x_{j}^{\prime}\right): x_{j}^{\prime} \leftarrow D_{j}^{\prime}\left(1^{\lambda}\right)\right\}
$$

We will show that:

$$
\begin{aligned}
& \left\{\left\langle s, \tilde{C}, \tilde{x}_{1}, \ldots \tilde{x}_{q}\right\rangle: x_{i} \leftarrow D_{i}\left(1^{\lambda}\right),(\tilde{C}, s) \leftarrow \mathrm{GC} \cdot \operatorname{circ}\left(1^{\lambda}, C\right), \tilde{x}_{i} \leftarrow \mathrm{GC} \cdot \operatorname{inp}\left(x_{i}, s\right)\right\} \\
& \stackrel{\operatorname{comp}}{\approx}\left\{\left\langle s, \tilde{C}, \tilde{x}_{1}^{\prime}, \ldots \tilde{x}_{q}^{\prime}\right\rangle: x_{i}^{\prime} \leftarrow D_{i}^{\prime}\left(1^{\lambda}\right),(\tilde{C}, s) \leftarrow \mathrm{GC} \cdot \operatorname{circ}\left(1^{\lambda}, C\right), \tilde{x}_{i}^{\prime} \leftarrow \mathrm{GC} \cdot \operatorname{inp}\left(x_{i}^{\prime}, s\right)\right\}
\end{aligned}
$$

Since we consider public input garbling (by including $s$ in the distributions), we can rely on a simple hybrid argument to show that the above holds as long as for each $i \in[q]$ :

$$
\left\langle s, \tilde{C}, \tilde{x}_{i}\right\rangle \stackrel{\text { comp }}{\approx}\left\langle s, \tilde{C}, \tilde{x}_{i}^{\prime}\right\rangle
$$

In particular, given $s$, we can efficiently sample the values $x_{j} \leftarrow D_{j}\left(1^{\lambda}\right), \tilde{x}_{j} \leftarrow \mathrm{GC}$.inp $\left(x_{j}, s\right)$ as well as $x_{j}^{\prime} \leftarrow D_{j}^{\prime}\left(1^{\lambda}\right), \tilde{x}_{j}^{\prime} \leftarrow \mathrm{GC}$.inp $\left(x_{j}^{\prime}, s\right)$ for all $j \neq i$ ourselves.

To show (1), we define the following hybrid distributions:

- $\mathrm{Hyb}_{0}$ : This is the distribution $\left\langle s, \tilde{C}, \tilde{x}_{i}\right\rangle$. Recall that $s=\left(\sigma, \mathrm{pk}_{1}, \mathrm{pk}_{2}\right), \tilde{C}=\mathcal{O}\left(C^{*}\right)$, where $\left.C^{*}=C^{*}\left[\sigma, \mathrm{pk}_{1}, \mathrm{pk}_{2}, 1, \mathrm{sk}_{1}, u=\perp, v=\perp\right]\right)$, and $\tilde{x}_{i}=\left(c_{1}, c_{2}, \pi\right)$.

- $\mathrm{Hyb}_{1}$ : In this hybrid, we switch the real proof to a simulated one for the statement st $=$ $\left(\mathrm{pk}_{1}, \mathrm{pk}_{2}, c_{1}, c_{2}\right)$. In particular, we now choose $(\sigma, \tau) \leftarrow S_{1}\left(1^{\lambda}\right.$, st $)$ to be a simulated CRS and $\pi \leftarrow S_{2}(\sigma, \tau, \mathrm{st})$ where $\left(S_{1}, S_{2}\right)$ are the simulators of the NIZK scheme. We claim:

$$
\mathrm{Hyb}_{0} \stackrel{\text { comp }}{\approx} \mathrm{Hyb}_{1}
$$

This follows directly from the computational zero-knowledge property of the NIZK П. 
- $\mathrm{Hyb}_{2}$ : In this hybrid, we encrypt $c_{2} \leftarrow \operatorname{Enc}\left(\mathrm{pk}_{2}, \overline{0}\right)$ in the second ciphertext, where $\overline{0}$ is the same length as $x_{i}$. The first ciphertext $c_{1}$ still encrypts $x_{i} \leftarrow D_{i}\left(1^{\lambda}\right)$. We claim:

$$
\mathrm{Hyb}_{1} \stackrel{\text { comp }}{\approx} \mathrm{Hyb}_{2}
$$

This follows directly from the semantic security of the encryption scheme. Notice that $\mathrm{sk}_{2}$ does not appear anywhere in either hybrid.

- $\mathrm{Hyb}_{3}$ : In this hybrid, we switch the circuit being obfuscated from $C_{1}^{*}=C^{*}\left[\sigma, \mathrm{pk}_{1}, \mathrm{pk}_{2}, 1, \mathrm{sk}_{1}, u=\right.$ $\perp, v=\perp]$ to $C_{2}^{*}=C^{*}\left[\sigma, \mathrm{pk}_{1}, \mathrm{pk}_{2}, 2, \mathrm{sk}_{2}, u=\left(c_{1}, c_{2}, \pi\right), v=y_{i}\right]$ where $y_{i}=C\left(x_{i}\right)$. Notice $C_{2}^{*}$ now uses $\mathrm{sk}_{2}$ to decrypt but has the values $u, v$ hard-coded. We claim that $C_{1}^{*}, C_{2}^{*}$ are functionally equivalent: for any input $u^{\prime}=\left(c_{1}^{\prime}, c_{2}^{\prime}, \pi^{\prime}\right)$ such that $u^{\prime} \neq u$ and $\pi^{\prime}$ verifies, the ciphertexts $\left(c_{1}^{\prime}, c_{2}^{\prime}\right)$ must encrypt the same message (by the statistical-simulation-soundness of the NIZK) and hence $C_{1}^{*}\left(u^{\prime}\right)=C_{2}^{*}\left(u^{\prime}\right)$. On the other hand, for input $u$, we have $C_{1}^{*}(u)=C_{2}^{*}(u)=y_{i}$. We claim:

$$
\mathrm{Hyb}_{2} \stackrel{\text { comp }}{\approx} \mathrm{Hyb}_{3}
$$

This follows directly from the indistinguishability obfuscation property, by noting the $C_{1}^{*}$ and $C_{2}^{*}$ are functionally equivalent.

- $\mathrm{Hyb}_{4}$ : In this hybrid, we also encrypt $c_{1} \leftarrow \operatorname{Enc}\left(\mathrm{pk}_{1}, \overline{0}\right)$ in the first ciphertext, where $\overline{0}$ is the same length as $x_{i}$. We claim:

$$
\mathrm{Hyb}_{3} \stackrel{\text { comp }}{\approx} \mathrm{Hyb}_{4}
$$

This follows directly from the semantic security of the encryption scheme. Notice that sk does not appear anywhere in either hybrid.

- $\mathrm{Hyb}_{5}$ : In this hybrid, we switch the hard-coded value in $C^{*}\left[\sigma, \mathrm{pk}_{1}, \mathrm{pk}_{2}, 2, \mathrm{sk}_{2}, u, v\right]$ from $v=y_{i}$ to $v=y_{i}^{\prime}=C\left(x_{i}^{\prime}\right)$ where $x_{i}^{\prime} \leftarrow D_{i}^{\prime}\left(1^{\lambda}\right)$. We claim:

$$
\mathrm{Hyb}_{4} \stackrel{\text { comp }}{\approx} \mathrm{Hyb}_{5}
$$

This follows from the condition that $C\left(x_{i}\right) \stackrel{\text { comp }}{\approx} C\left(x_{i}^{\prime}\right)$. Notice that no other information about $x_{i}, x_{i}^{\prime}$ other than $y_{i}=C\left(x_{i}\right), y_{i}^{\prime}=C\left(x_{i}^{\prime}\right)$ appears in either hybrid.

- $\mathrm{Hyb}_{6}$ to $\mathrm{Hyb}_{10}$ : These are the same as $\mathrm{Hyb}_{4}$ through $\mathrm{Hyb}_{0}$ but using $\left(x_{i}^{\prime}, y_{i}^{\prime}\right)$ in place of $\left(x_{i}, y_{i}\right)$. Notice that $\mathrm{Hyb}_{10}$ is just the distribution $\left\langle s, \tilde{C}, \tilde{x}_{i}^{\prime}\right\rangle$. By symmetry, we get:

$$
\mathrm{Hyb}_{5} \stackrel{\text { comp }}{\approx} \mathrm{Hyb}_{10}
$$

Combining the above, we get $\mathrm{Hyb}_{0} \stackrel{\text { comp }}{\approx} \mathrm{Hyb}_{10}$, which proves equation (1) and the theorem follows.

\section{F.3 Proof of Theorem 4.4}

Proof of Theorem 4.4. Let us fix some polynomial size input specification in $=\left((N, n, m, t), D, P,\left\langle x_{i}\right\rangle\right)$ with corresponding output specification out $=\left((N, n, m, t), P,\left\langle y_{i}\right\rangle\right)$ where $i=1, \ldots, q$.

The simulator GR.sim gets input $\left(1^{\lambda}\right.$, out). It begins by garbling a dummy database $\left(\tilde{D}^{\prime}, k\right) \leftarrow$ GR.data $\left(1^{\lambda}, 0^{N}\right)$. It then constructs the circuit $C=C[P, N, n, m, t, \lambda]$ and applies the circuitgarbling procedure to get $(\tilde{C}, s) \leftarrow \mathrm{GC} \cdot \operatorname{circ}\left(1^{\lambda}, C\right)$. Next, for every $y_{i}$ the simulator chooses a 
uniformly random $r_{i} \leftarrow\{0,1\}^{2 \lambda}$, sets $w_{i}^{\prime}=\left(k, r_{i}, 0^{n}, \psi=0, y_{i}, i\right)$ and $\tilde{w}_{i}{ }^{\prime} \leftarrow \operatorname{GC} \cdot \operatorname{inp}\left(w_{i}^{\prime}, s\right)$. The output of the simulator GR.sim consists of

$$
\left(\tilde{D}^{\prime}, \tilde{C}, \quad \tilde{w}_{1}^{\prime}, \ldots, \tilde{w}_{q}^{\prime}\right) \text {. }
$$

We next prove indistinguishability between the real and simulated outputs. Notice that

$$
\operatorname{Real}[\text { in, } \lambda]=\left(\tilde{D}, \tilde{C}, \quad \tilde{w}_{1}, \ldots, \tilde{w}_{q}\right)
$$

where $(\tilde{D}, k) \leftarrow \mathrm{GR} \cdot \operatorname{data}\left(1^{\lambda}, D\right),(\tilde{C}, s) \leftarrow \mathrm{GC} \cdot \operatorname{circ}\left(1^{\lambda}, C\right)$, and we set $w_{i}=\left(k, r_{i}, x_{i}, \psi=1,0^{m}, i\right)$ for random $r_{i} \leftarrow\{0,1\}^{2 \lambda}$ and $\tilde{w}_{i} \leftarrow \mathrm{GC} \cdot \operatorname{inp}\left(w_{i}^{\prime}, s\right)$.

Claim F.2. Denote $C=C[P, N, n, m, t, \lambda]$. If the scheme GR1 = (GR1.prog, GR1.inp, GR1.eval) satisfies one-time security with persistent memory (Definition 4.2) then

$$
\left(\tilde{D}, C\left(w_{1}\right), \ldots, C\left(w_{q}\right)\right) \stackrel{\text { comp }}{\approx}\left(\tilde{D}^{\prime}, C\left(w_{1}^{\prime}\right), \ldots, C\left(w_{q}^{\prime}\right)\right) \text {. }
$$

Proof. Note that $C\left(w_{i}\right)=\left(\tilde{P}_{i}, \tilde{x}_{i}\right)$ and $C\left(w_{i}^{\prime}\right)=\left(\tilde{P}_{i}, \tilde{x}_{i}^{\prime}\right)$ where each $\tilde{P}_{i}$ is a freshly garbled version of the program $P^{+}, \tilde{x}_{i}$ is a garbled version of the input $\left(x_{i}, \psi=1,0^{m}\right)$, and $\tilde{x}_{i}^{\prime}$ is a garble version of the input $\left(0^{n}, \psi=0, y_{i}\right)$. The one-time simulation-security of the underlying GR1 scheme implies that:

$$
\left(\tilde{D},\left\langle\tilde{P}_{i}, \tilde{x}_{i}\right\rangle_{i \in[q]}\right) \stackrel{\mathrm{comp}}{\approx} \mathrm{GR} 1 \cdot \operatorname{Sim}\left(1^{\lambda}, \text { out }\right) \stackrel{\mathrm{comp}}{\approx}\left(\tilde{D}^{\prime},\left\langle\tilde{P}_{i}, \tilde{x}_{i}^{\prime}\right\rangle_{i \in[q]}\right) .
$$

This proves the claim.

We now conclude that the simulated and real distributions are the indistinguishable:

$$
\left(\tilde{D}^{\prime}, \tilde{C},\left\langle\tilde{w}_{i}^{\prime}\right\rangle_{i \in[q]}\right) \stackrel{\operatorname{comp}}{\approx}\left(\tilde{D}, \tilde{C},\left\langle\tilde{w}_{i}\right\rangle_{i \in[q]}\right)
$$

This follows by the above claim in conjunction with the definition of correlated distributional indistinguishability on the circuit garbling scheme. We think of $\tilde{D}=$ aux and $\tilde{D}^{\prime}=$ aux $x^{\prime}$ as auxiliary input.

\section{F.4 Proof of Theorem 4.8}

Proof of Theorem 4.8. Let $C$ be a circuit (ensemble) and $D$ and $D^{\prime}$ be efficiently samplable input distributions (ensembles) such that it hold that

$$
\left\langle C\left(x_{1}^{0}\right), \ldots, C\left(x_{n}^{0}\right), \operatorname{aux}_{0}\right\rangle \stackrel{\operatorname{comp}}{\approx}\left\langle C\left(x_{1}^{1}\right), \ldots, C\left(x_{n}^{1}\right), \operatorname{aux}_{1}\right\rangle .
$$

where $\left\langle x_{1}^{0}, \ldots, x_{n}^{0}\right.$, aux $\left._{0}\right\rangle \leftarrow D\left(1^{\lambda}\right),\left\langle x_{1}^{1}, \ldots, x_{n}^{1}\right.$, aux 1$\rangle \leftarrow D^{\prime}\left(1^{\lambda}\right)$. We will show that

$$
\left\langle\tilde{C}, \tilde{x}_{1}^{0}, \ldots \tilde{x}_{n}^{0}, \operatorname{aux}_{0}\right\rangle \stackrel{\operatorname{comp}}{\approx}\left\langle\tilde{C}, \tilde{x}_{1}^{1}, \ldots \tilde{x}_{n}^{1}, \operatorname{aux}_{1}\right\rangle
$$

where $(\tilde{C}, s) \leftarrow \mathrm{GC} \cdot \operatorname{circ}\left(1^{\lambda}, C\right), \tilde{x}_{i}^{0} \leftarrow \mathrm{GC} \cdot \operatorname{inp}\left(x_{i}^{0}, s\right), \tilde{x}_{i}^{1} \leftarrow \mathrm{GC} \cdot \operatorname{inp}\left(x_{i}^{1}, s\right)$.

We consider the following sequence of hybrid distributions to show the indistinguishability of the above distributions:

- $\mathrm{Hyb}_{0}$ : This is the distribution $\left\langle\tilde{C}, \tilde{x}_{1}^{0}, \ldots \tilde{x}_{n}^{0}\right.$, aux $\rangle$, where $\tilde{C} \leftarrow \mathcal{O}\left(C^{*}\right)$ for $C^{*}:=C^{*}\left[\sigma, \mathrm{pk}_{0}, \mathrm{pk}_{1}, 0, \mathrm{sk}_{0}\right]$, and $\tilde{x}_{i}^{0}=\left(c_{0}^{i}, c_{1}^{i}, \pi^{i}\right)$ where $c_{b}^{i} \leftarrow \operatorname{Enc}\left(\mathrm{pk}_{b}, x_{i}^{0} ; r_{b}^{i}\right), \pi^{i} \leftarrow P\left(\sigma,\left(\mathrm{pk}_{0}, \mathrm{pk}_{1}, c_{0}^{i}, c_{1}^{i}\right),\left(r_{0}^{i}, r_{1}^{i}\right)\right)$ for all $1 \leq i \leq n$. 
- $\mathrm{Hyb}_{1}$ : In this hybrid, we compute the proofs $\pi^{i}$ using the NIZK simulators $\left(S_{1}, S_{2}\right)$, i.e., $(\sigma, \tau) \leftarrow S_{1}\left(1^{\lambda}\right), \pi^{i} \leftarrow S_{2}\left(\sigma, \tau, c_{0}^{i}, c_{1}^{i}\right)$ for $1 \leq i \leq n$.

The indistinguishability $\mathrm{Hyb}_{0} \stackrel{\text { comp }}{\approx} \mathrm{Hyb}_{1}$ follows from the computational zero-knowledge of property of the NIZK $\Pi$.

- $\mathrm{Hyb}_{2}$ : In this hybrid, we also change the way we compute the garbled inputs by setting $c_{1}^{i} \leftarrow \operatorname{Encrypt}\left(\mathrm{pk}_{1}, x_{i}^{\prime}\right)$ to be an encryption of $x_{i}^{1}$ rather than $x_{i}^{0}$, for all $1 \leq i \leq n$, . The NIZK CRS and proofs $\pi_{i}$ are still computed using the NIZK simulator.

The indistinguishability $\mathrm{Hyb}_{1} \stackrel{\text { comp }}{\approx} \mathrm{Hyb}_{2}$ follows from the semantic security of the encryption scheme $\mathcal{P} \mathcal{K} \mathcal{E}$ since sk $_{1}$ does not appear anywhere in the garbled circuit.

- $\mathrm{Hyb}_{3}$ : In this hybrid, we change the way we compute the garbled circuit by obfuscating $C^{*}\left[\sigma, \mathrm{pk}_{0}, \mathrm{pk}_{1}, b=1, \mathrm{sk}_{1}\right]$ with the bit $b=1$ and the secret key sk $\left(\right.$ instead of $b=0$ and $\mathrm{sk}_{0}$ ). That is, we set $\tilde{C}^{\prime} \leftarrow \mathcal{O}\left(C^{*}\left[\sigma, \mathrm{pk}_{0}, \mathrm{pk}_{1}, b=1, \mathrm{sk}_{1}\right]\right)$. We also change the auxiliary input from aux $_{0}$ to aux 1 We claim that:

$$
\mathrm{Hyb}_{2} \stackrel{\text { comp }}{\approx} \mathrm{Hyb}_{3} \text {. }
$$

This follows from the strong differing-inputs obfuscation property. Firstly, we claim that $(\mathcal{C}$, Sam $)$ is a relevant differing-input family where $\mathcal{C}=\left\{C^{*}\left[\sigma, \mathrm{pk}_{0}, \mathrm{pk}_{1}, b, \mathrm{sk}\right]\right\}$ and

$$
\left(C_{0}=C^{*}\left[\sigma, \mathrm{pk}_{0}, \mathrm{pk}_{1}, b=0, \mathrm{sk}_{0}\right], C_{1}=C^{*}\left[\sigma, \mathrm{pk}_{0}, \mathrm{pk}_{1}, b=1, \mathrm{sk}_{1}\right], \tilde{x}_{1}, \ldots, \tilde{x}_{n}, \mathrm{aux}_{0}, \mathrm{aux}_{1}\right) \leftarrow \operatorname{Sam}^{\lambda}\left(1^{\lambda}\right)
$$

is defined by sampling $\left(\mathrm{pk}_{0}, \mathrm{sk}_{0}\right) \leftarrow \operatorname{Setup}\left(1^{\lambda}\right),\left(\mathrm{pk}_{1}, \mathrm{sk}_{1}\right) \leftarrow \operatorname{Setup}\left(1^{\lambda}\right),(\sigma, \tau) \leftarrow S_{1}\left(1^{\lambda}\right)$, $\left\langle x_{1}^{0}, \ldots, x_{n}^{0}\right.$, aux $\left._{0}\right\rangle \leftarrow D\left(1^{\lambda}\right),\left\langle x_{1}^{1}, \ldots, x_{n}^{1}\right.$, aux $\left._{1}\right\rangle \leftarrow D^{\prime}\left(1^{\lambda}\right)$ and setting $\tilde{x}_{i}=\left(c_{0}^{i}, c_{1}^{i}, \pi^{i}\right)$ where $c_{b}^{i} \leftarrow \operatorname{Enc}\left(\mathrm{pk}_{b}, x_{i}^{b}\right)$ and $\pi^{i} \leftarrow S_{2}\left(\sigma, \tau, c_{0}^{i}, c_{1}^{i}\right)$.

This follows since

$$
\left(\left\langle\tilde{x}_{i}, C_{0}\left(\tilde{x}_{i}\right)=x_{i}^{0}\right\rangle_{i \in[q]}, \operatorname{aux}_{0}\right) \stackrel{\text { comp }}{\approx}\left(\left\langle\tilde{x}_{i}, C_{1}\left(\tilde{x}_{i}\right)=x_{i}^{1}\right\rangle_{i \in[q]}, \operatorname{aux}_{1}\right)
$$

by assumption on the distributions $D, D^{\prime}$. Furthermore, coming up with an input $\tilde{x} \notin$ $\left\{\tilde{x}_{1}, \ldots, \tilde{x}_{n}\right\}$ such that $C_{0}(\tilde{x}) \neq C_{1}(\tilde{x})$ requires coming up with a valid NIZK proof for a new false statement, which is hard by the simulation-soundness security of the NIZK even given $C_{0}, C_{1},\left\langle\tilde{x}_{i}\right\rangle$, aux 0 , aux 1 .

Now that we showed $(\mathcal{C}$, Sam $)$ is a relevant differing-input family, we can rely on sdiO security of the obfuscator $\mathcal{O}$ to argue that $\mathrm{Hyb}_{2} \stackrel{\text { comp }}{\approx} \mathrm{Hyb}_{3}$. In particular one cannot distinguish $\left(\mathcal{O}\left(1^{\lambda}, C_{0}\right),\left\langle\tilde{x}_{i}^{\prime}\right\rangle_{i \in[q]}\right.$, aux 0$)$ from $\left(\mathcal{O}\left(1^{\lambda}, C_{1}\right),\left\langle\tilde{x}_{i}^{\prime}\right\rangle_{i \in[q]}\right.$, aux 1$)$ where $\tilde{x}_{i}^{\prime}$ are chosen as in hybrid 2.

- $\mathrm{Hyb}_{4}$ to $\mathrm{Hyb}_{6}$ : These hybrid are symmetric to the changes introduced by $\mathrm{Hyb}_{1}$ to $\mathrm{Hyb}_{3}$ in a slightly different order. In $\mathrm{Hyb}_{4}$ we change the encryptions to $c_{0}^{i}=\operatorname{Encrypt}\left(\mathrm{pk}_{0}, x_{i}^{1}\right)(\operatorname{semantic}$ security), in $\mathrm{Hyb}_{5}$ we switch to honestly generated NIZK proofs $\pi^{i}$ (ZK property) and finally in $\mathrm{Hyb}_{6}$ we switch back to an obfuscation of the circuit $C_{0}=C^{*}\left[\sigma, \mathrm{pk}_{0}, \mathrm{pk}_{1}, b=0, \mathrm{sk}_{0}\right]$ (sdiO). The last $\mathrm{Hyb}_{6}$ is just the distribution $\left\langle\tilde{C}, \tilde{x}_{1}^{1}, \ldots \tilde{x}_{n}^{1}\right\rangle$. By the same arguments as described above, we get

$$
\mathrm{Hyb}_{3} \stackrel{\text { comp }}{\approx} \mathrm{Hyb}_{6} \text {. }
$$

Combining the above, we get that $\mathrm{Hyb}_{0} \stackrel{\text { comp }}{\approx} \mathrm{Hyb}_{6}$, which completes the proof of the theorem. 